\title{
Experimental study of the Al-Cu-Zn ternary phase diagram
}

\author{
Ondrej Zobac ${ }^{1,2, *}$ (D), Ales Kroupa ${ }^{2}$, and Klaus W. Richter ${ }^{1}$ \\ ${ }^{1}$ Department of Inorganic Chemistry - Functional Materials, University of Vienna, Währinger Straße 42, 1090 Vienna, Austria \\ ${ }^{2}$ Institute of Physics of Materials, The Czech Academy of Sciences, Zizkova 22, 61600 Brno, Czech Republic
}

Received: 10 February 2020

Accepted: 15 April 2020

Published online:

30 April 2020

(C) The Author(s) 2020

\begin{abstract}
The phase diagram of the $\mathrm{Al}-\mathrm{Cu}-\mathrm{Zn}$ ternary system was re-investigated experimentally. The current study was designed to contribute to a better description of those parts of the phase diagram, which are disputed in the current scientific literature. Mutual relations in the family of ternary intermetallic phases $\tau$ with cubic, rhombohedral and modulated structure at temperatures $400{ }^{\circ} \mathrm{C}$ and $550{ }^{\circ} \mathrm{C}$ were described. The phase relation between the $\gamma$ and $\gamma^{\prime}$ phases was studied at different temperatures. A two-phase field between $\gamma$ and $\gamma^{\prime}$ was observed below $400{ }^{\circ} \mathrm{C}$, while the transition appears to be second order at higher temperatures. A vertical section between $\gamma$ and $\gamma^{\prime}$ phases in $\mathrm{Cu}-$ $\mathrm{Zn}$ and $\mathrm{Al}-\mathrm{Cu}$ and four isothermal sections at $400{ }^{\circ} \mathrm{C}, 550{ }^{\circ} \mathrm{C}, 700{ }^{\circ} \mathrm{C}$ and $820^{\circ} \mathrm{C}$, respectively, were constructed.
\end{abstract}

\section{Introduction and literature reviewIntroduction}

The Al-Cu-Zn ternary system has been investigated intensively in the past because of its technical importance for developing of light-weight alloys. However, several areas of the phase diagram are still not well understood and discussed intensively. Our work is focused on several doubtful regions of the phase diagram, which have not yet been satisfactorily resolved in the scientific literature, and on the divergent results from the experimental and theoretical publications [1, 2]. Major topics were the extensions of the $\gamma$ and $\gamma^{\prime}$ solid solution phases (with primitive and base-centered brass structures, respectively) and their mutual relation in the whole concentration and temperature range. We also studied the crystal structure and homogeneity ranges of the reported ternary phases $\tau$ and $\tau^{\prime}$ and constructed the isothermal sections of the phase diagram $\mathrm{Al}-\mathrm{Cu}-$ $\mathrm{Zn}$ based on our new experimental results at $400{ }^{\circ} \mathrm{C}$, $550{ }^{\circ} \mathrm{C}, 700{ }^{\circ} \mathrm{C}$ and $820^{\circ} \mathrm{C}$ as well as the vertical section describing the mutual relation between $\gamma$ and $\gamma^{\prime}$ phases.

\section{Literature review}

Detailed literature reviews of all binary subsystems are described in recent literature. The experimental description of the Al-Cu phase diagram is published in our previous work [3]. Liang et al. [4] published a

Address correspondence to E-mail: zobac@ipm.cz 
theoretical modeling of the $\mathrm{Al}-\mathrm{Cu}$ phase diagram based on CALPHAD approach.

The Al-Zn binary phase diagram was assessed by Murray [5] and reported by Massalski [6]. Dinsdale et al. [7] have done the most recent theoretical modeling of the $\mathrm{Al}-\mathrm{Zn}$ phase diagram, using the third generation of unary data. Theoretical modeling using the commonly used SGTE unary database [8] was published by Mathon et al. [9].

The assessed $\mathrm{Cu}-\mathrm{Zn}$ binary phase diagram was published by Miodownik [10]. Liang et al. [11] published the most recent theoretically modeled binary phase diagram $\mathrm{Cu}-\mathrm{Zn}$. Figure $1 \mathrm{a}-\mathrm{c}$ show redrawn binary experimental phase diagrams of the relevant subsystems.

Ghosh et al. [1] published a review of the experimental studies on the $\mathrm{Al}-\mathrm{Cu}-\mathrm{Zn}$ phase diagram available up to the year 2002. The experimental ternary phase diagram $\mathrm{Al}-\mathrm{Cu}-\mathrm{Zn}$ provided in [1] can be generally accepted except for the disagreement in the proposed phase equilibria between the $\gamma$ and $\gamma^{\prime}$ phases in comparison with the detailed analysis of existing experimental studies presented by Liang and Schmid-Fetzer [2]. These authors also published the most recent theoretical assessment based on this analysis in the same paper [2]. The main difference exists for mutual relation between the two closely related $\gamma\left(\mathrm{Al}_{4} \mathrm{Cu}_{9}\right)$ and $\gamma^{\prime}\left(\mathrm{Cu}_{5} \mathrm{Zn}_{8}\right)$ phases and ternary $\tau$ and $\tau^{\prime}$ phases which will be discussed later. Most of the original references from the years 1905-2002 evaluated in Ghosh et al. [1] are not duplicated here.

Liang and Chang [12] published an overall thermodynamic description based on the CALPHAD approach. Two isothermal sections at $500{ }^{\circ} \mathrm{C}$ and $700{ }^{\circ} \mathrm{C}$ and a liquidus projection were published in this paper. Only one ternary intermetallic compound $\tau$ (approx. formula $\mathrm{Al}_{3} \mathrm{Cu}_{5} \mathrm{Zn}_{2}$ ) was theoretically modeled in this system by Liang and Chang [12]. Despite the fact that the experimentally established homogeneity range of the $\tau$ phase is highly temperature dependent, Liang and Schmid-Fetzer [2] modeled the $\tau$ phase as a linear phase with constant composition $x(\mathrm{Zn})=0.10$. It was found earlier that the family of $\tau$-phases appears in two modifications, cubic B2-type $\tau$ phase and a structurally related rhombohedral $\tau^{\prime}$ phase [13]. The $\tau^{\prime}$ phase was not included into Liang's [2, 12] thermodynamic description because the composition range and thermal stability had not yet been reasonably determined.
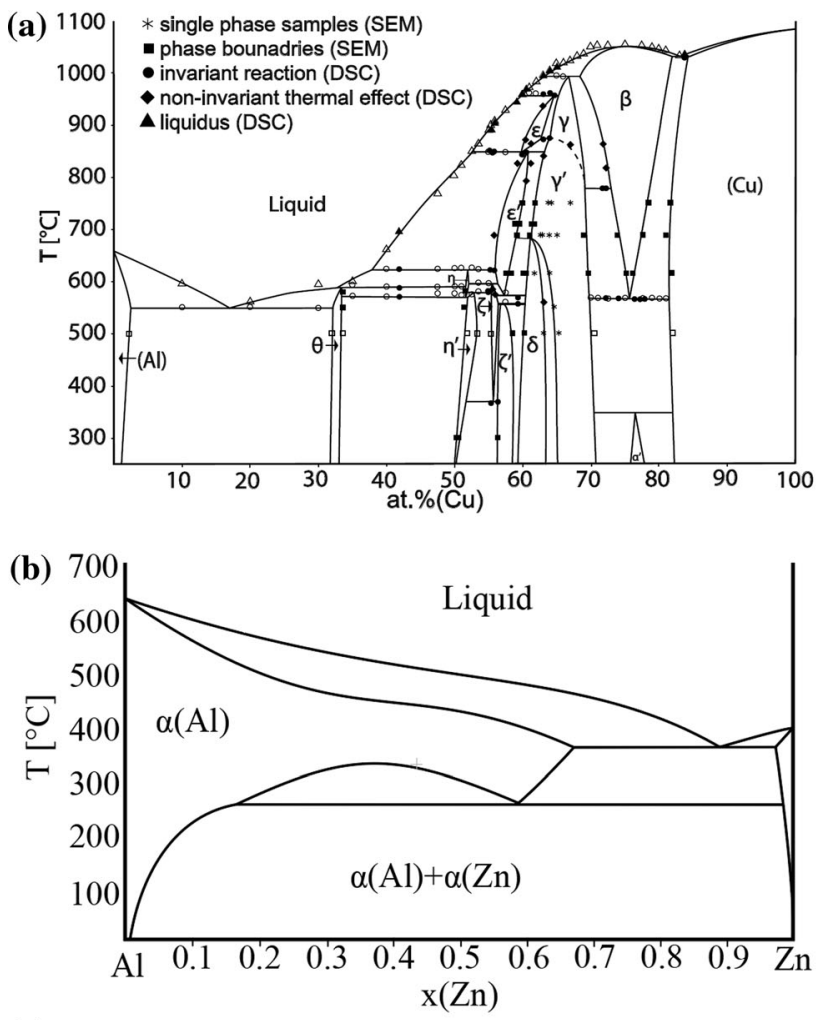

(c)

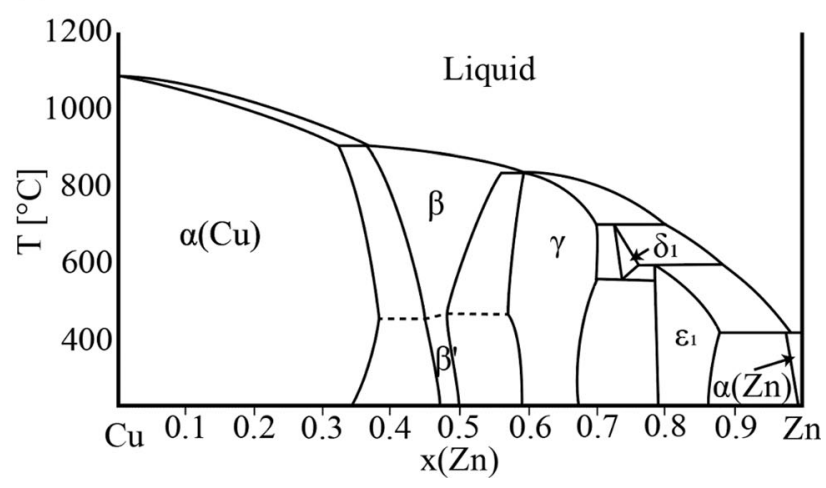

Figure 1 Binary phase diagram of subsystem. a Al- $\mathrm{Cu}[3]$, b Al$\mathrm{Zn}$ [6], c $\mathrm{Cu}-\mathrm{Zn}$ [6].

The continuous solid solutions were modeled between some binary intermetallic phases in this system. The $\beta$ (BCC_A2) phase exists in both Al-Cu and $\mathrm{Cu}-\mathrm{Zn}$ systems and the continuous solid solution was modeled in [12]. The situation is more complicated in the case of the family of $\gamma$-phases. The $\gamma$ phase region forms continuous solid solution in all experimentally assessed isothermal sections from $350{ }^{\circ} \mathrm{C}$ to $700{ }^{\circ} \mathrm{C}$ [1], from the $\mathrm{Al}-\mathrm{Cu}$ side, where the low temperature $\gamma^{\prime}\left(\mathrm{Al}_{4} \mathrm{Cu}_{9}\right)$ with the Pearson symbol cP52 exists in the binary $\mathrm{Al}-\mathrm{Cu}$ system, to the $\mathrm{Cu}-\mathrm{Zn}$ 
side where the $\gamma\left(\mathrm{Cu}_{5} \mathrm{Zn}_{8}\right)$ phase with the Pearson symbol cI52 is stable.

The first results showing continuous $\gamma$ phase region between $\mathrm{Al}-\mathrm{Cu}$ and $\mathrm{Cu}-\mathrm{Zn}$ binaries were presented by Bauer and Hansen [14]. They constructed several isothermal sections of $\mathrm{Al}-\mathrm{Cu}-\mathrm{Zn}$ ternary system in the $\mathrm{Cu}$-rich corner in temperature range $410-800{ }^{\circ} \mathrm{C}$. They proposed the continuous solid solution of the $\gamma$ phase based on their experimental results. Koster and Moeller [13] constructed an isothermal section at $350{ }^{\circ} \mathrm{C}$ and confirmed the continuous solubility of the $\gamma$ phase. Ashirimbetov et al. [15] reported two isothermal sections at $20^{\circ} \mathrm{C}$ and $350{ }^{\circ} \mathrm{C}$. The results showed the solid solubility of $\mathrm{Al}$ in $\gamma$ at $20^{\circ} \mathrm{C}$ and $350{ }^{\circ} \mathrm{C}$ are about 3.5 and 7.0 wt \% (2.2 and 5.5 at.\%), respectively, and solubility of $\mathrm{Zn}$ in $\gamma^{\prime}$ at the same temperatures about $30 \mathrm{wt} \%$. They detected a wide two-phase region between $\gamma$ and $\gamma^{\prime}$ at room temperature and $350{ }^{\circ} \mathrm{C}$. They predicted that these two different $\gamma$ phases might be miscible at higher temperatures where the high temperature modification of the $\gamma$ phase exists in the Al-Cu system with the same crystal structure as in the $\mathrm{Cu}-\mathrm{Zn}$ system. Also Liang and Schmid-Fetzer [2] did not accept the continuous solid solution between these crystallographically slightly different types of $\gamma$ phases in their theoretical work. They described this decision in detail in the text of their paper. Nevertheless, they did not run any experimental study to confirm their conclusions.

With respect to previously mentioned result discrepancies, the aim of our study of the Al-Cu-Zn ternary system was to experimentally solve the question of solubilities between the $\gamma^{\prime}$ and $\gamma$ phases and a detailed crystallography and compositional and temperature stability of $\tau$ and $\tau^{\prime}$ phases.

The current available information for all solid phases of the system is summarized in Table 1. The abbreviated phase names in the form of Greek letters used in the text and phase diagrams are provided in the first column. Other common phase names used in the literature [1-3] are shown in column 2. The Pearson symbol and structure type (columns 3 and 4) are taken from the Materials Science International Team (MSIT) report [16]. Temperature stability of binary phases is taken from relevant phase diagrams $[3,6]$. Temperature stability of ternary phase $\tau$ (in our text is equal to $\tau_{\mathrm{c}}$ ) from Ghosh et al. [1] and ternary phases $\tau_{\mathrm{i}}$ and $\tau_{\mathrm{r}}$ (which corresponds to $\tau^{\prime}$ ) is based on our new results.

\section{Experimental}

The overall compositions of experimental samples were selected in order to address the unsolved questions in the experimental phase diagrams as mentioned above. Furthermore, it was our aim to determine complete isothermal phase equilibria in four isothermal sections. The prepared samples were analyzed and characterized by different static and dynamic analytical methods (SEM-EDX, DTA, XRD).

\section{Sample preparation}

Samples were prepared from pure elements of $5 \mathrm{~N}$ purity. Any oxide presented in the copper was reduced by flowing of the $\mathrm{H}_{2}$ gas at $300{ }^{\circ} \mathrm{C}$ for $3 \mathrm{~h}$. The alloys were re-melted at $950{ }^{\circ} \mathrm{C}$ several times in evacuated quartz glass ampoules in order to improve the homogenization of the material. Long-term annealing of the samples was performed at selected temperatures on material sealed in evacuated quartz glass ampoules. To prevent reaction of liquid $\mathrm{Al}$ with the Si from material of quartz glass, the samples with high content of Al-rich liquid were placed inside the corundum crucibles. These crucibles with samples were sealed in evacuated quartz glass ampoules. A conventional muffle resistance furnace was used for the heat treatment. Samples were long-term annealed to achieve state close to the thermodynamic equilibrium. Annealing time was selected with respect to annealing temperature. For the annealing temperature closer to the melting temperature, shorter annealing time is sufficient. Annealing was terminated by quenching of the samples into cold water from their annealing temperatures, and sample was prepared for further investigations. Annealing times and temperatures are given in Table 2 together with experimental results.

\section{Experimental phase diagram investigation}

A combination of dynamic and static methods was used for the investigation of the phase diagram. Phase equilibria, microstructure and chemical analysis of phases and overall compositions were performed by using scanning electron microscopy combined with energy dispersive X-ray spectroscopy (SEM-EDX), employing either a Zeiss Supra 55 VP instrument equipped with an energy dispersive detector for quantitative analysis or a similarly 
Table 1 Stable phases in Al-Cu-Zn ternary phase diagram and binary subsystems

\begin{tabular}{|c|c|c|c|c|}
\hline Phase name [This work] & Common names & Pearson symbol & Structure prototype & T. range $\left({ }^{\circ} \mathrm{C}\right)$ \\
\hline$\alpha(\mathrm{Al})$ & FCC_A1, Al & $c F 4$ & $\mathrm{Al}$ & $\leq 660.5$ \\
\hline$\alpha(\mathrm{Cu})$ & $\mathrm{FCC} A 1, \mathrm{Cu}$ & $c F 4$ & $\mathrm{Cu}$ & $\leq 1083$ \\
\hline$\alpha(\mathrm{Zn})$ & $\mathrm{HCP} \mathrm{Zn}, \mathrm{Zn}$ & $h P 2$ & $\mathrm{Mg}$ & \\
\hline$\theta$ & $\theta, \overline{\mathrm{Al}_{2}} \mathrm{Cu}$ & $t I 12$ & $\mathrm{Al}_{2} \mathrm{Cu}$ & $\leq 590.5$ \\
\hline$\eta$ & $\eta_{1} \_\mathrm{AlCu}$, Eta $\mathrm{HT}$ & $o P 16 / o C 16$ & n.a. & $573.9-624.5$ \\
\hline$\eta^{\prime}$ & $\eta_{2}{ }_{-} \mathrm{AlCu}$, Eta $\mathrm{LT}$ & $m C 20$ & $\mathrm{AlCu}$ & $\leq 574.5$ \\
\hline$\zeta$ & $\zeta_{2}, \mathrm{Al}_{3} \mathrm{Cu}_{4-\delta}$ & $\operatorname{Imm} 2$ & $\mathrm{Al}_{3} \mathrm{Cu}_{4-\delta}$ & $373-597$ \\
\hline$\zeta^{\prime}$ & $\zeta_{1}, \mathrm{Al}_{3} \mathrm{Cu}_{4}$ & Fmm2 & $\mathrm{Al}_{3} \mathrm{Cu}_{4}$ & $\min .300-560.5$ \\
\hline$\varepsilon$ & $\varepsilon_{1} \mathrm{AlCu}$, epsilon $\mathrm{HT}$ & cubic? & n.a. & $959-846$ \\
\hline$\varepsilon^{\prime}$ & $\varepsilon_{2} \mathrm{AlCu}$, epsilon $\mathrm{LT}$ & $h P 4$ & $\mathrm{NiAs}$ & $846-568.5$ \\
\hline$\delta$ & $\delta, \mathrm{Al}_{5} \mathrm{Cu}_{8}$ & $h R 52$ & $\mathrm{Al}_{4} \mathrm{Cu}_{9}(\mathrm{r})$ & $\leq 680$ \\
\hline$\gamma$ & $\gamma_{0}, \gamma_{-} \mathrm{CuZn}, \gamma_{-}$brass & cI52 & $\mathrm{Cu}_{5} \mathrm{Zn}_{8}$ & $991-779.6$ \\
\hline$\gamma^{\prime}$ & $\gamma_{1}, \gamma_{-} \mathrm{AlCu}, \gamma_{-} \mathrm{D} 8_{3}$ & $c P 52$ & $\mathrm{Al}_{4} \mathrm{Cu}_{9}$ & $\leq 873.5$ \\
\hline$\beta$ & BCC_A2 (AlCu) & $c I 2$ & $\mathrm{~W}$ & $1052-566.7$ \\
\hline$\alpha$ & $\alpha_{2 \_} \mathrm{AlCu}$, alpha_LT & n. a. & super structure based on $\mathrm{TiAl}_{3}$ & $\leq 360$ \\
\hline$\beta$ ' & BCC_B2, CuZn & $c P 2$ & $\mathrm{CsCl}$ & $\leq 468$ \\
\hline$\delta_{1}$ & $\mathrm{CuZn}_{3}^{-}$ & $h P 3$ & $\mathrm{CuZn}_{3}$ & $700-560$ \\
\hline$\varepsilon_{1}$ & $\mathrm{CuZn}_{4}$ & $h P 2$ & $\mathrm{Mg}$ & $\leq 598$ \\
\hline$\tau_{\mathrm{c}}$ & $\tau, \mathrm{Cu}_{5} \mathrm{Zn}_{2} \mathrm{Al}_{3}$ & $c P 2$ & $\mathrm{CsCl}$ & $\leq 740$ \\
\hline$\tau_{\mathrm{i}}$ & & & Incommensurate $\tau$ phase & $\leq 550^{*}$ \\
\hline$\tau_{\mathrm{r}}$ & $\tau^{\prime}$ & $h R 9$ & superstructure of $\mathrm{CsCl}$ & $<440^{*}$ \\
\hline
\end{tabular}

*Based on constructed isothermal sections in this work

equipped SEM JEOL JSM-6460. Identification of phases present in the long-term annealed samples was achieved using X-ray powder diffraction. The Bruker D8 diffractometer equipped with a highspeed position sensitive (PSD) detector (Lynxeye) was used in the $\theta / 2 \theta$ reflection setting. Rietveld refinements of selected diffraction patterns were performed with the Topas software [17]. Annealing temperature of the sample, overall composition, coexisting phases and phase compositions of the concrete phases in the equilibrium are listed in Table 2. Annealing temperature and number of the sample are listed in column 1 , and the annealing time is shown in column 2. Column 3 shows the overall composition measured by SEM-EDX area scans. Coexisting phases stable in the samples are listed in column 4; columns 5-7 show the composition of equilibrium phases existing in the samples measured by SEM-EDX in same order as the phases are mentioned in column 4.

Phase transition temperatures were measured using a high-temperature DTA (NETZSCH Pegasus $404 \mathrm{C}$ ) with samples placed in closed and evacuated quartz glass DTA ampoules. Closed ampoules were used to limit uncontrolled $\mathrm{Zn}$ evaporation during the measurement and contamination of the inner parts of the instrument. The DTA was calibrated using a set of pure metal standards having well-defined melting temperatures $(\mathrm{Sn}, \mathrm{Al}, \mathrm{Zn}, \mathrm{Cu}, \mathrm{Ag}, \mathrm{Au}$ ). Calibration was carried out under the same conditions as the experimental measurements. Three heating and cooling runs were performed for each sample; the thermal effects during the first heating run were not taken into account. Thermal analysis results for four samples situated in the section $\gamma^{\prime}-\gamma$ are listed in Table 3.

\section{Results and discussion}

By combining all experimental results listed in Tables 2 and 3, it was possible to draw complete isothermal sections of ternary phase diagram $\mathrm{Al}-\mathrm{Cu}-$ $\mathrm{Zn}$ at $400{ }^{\circ} \mathrm{C}, 550{ }^{\circ} \mathrm{C}, 700{ }^{\circ} \mathrm{C}$ and $820^{\circ} \mathrm{C}$. These sections are presented in Fig. 2. The shape of the phase boundaries and phase fields not defined by our own samples was drawn based on information from 
Table 2 Chemical composition of the long-term annealed representative sample

\begin{tabular}{|c|c|c|c|c|c|c|c|c|c|c|c|c|c|c|}
\hline \multirow[t]{2}{*}{$\mathrm{T}\left[{ }^{\circ} \mathrm{C}\right] \_$No. } & \multirow[t]{2}{*}{$\begin{array}{l}\text { Annealing time } \\
{[\mathrm{h}]}\end{array}$} & \multicolumn{3}{|c|}{$\begin{array}{l}\text { Overall composition } \\
\text { (at. \%) }\end{array}$} & \multirow[t]{2}{*}{ Coexist. phases } & \multicolumn{3}{|c|}{ Phase 1 (at. \%) } & \multicolumn{3}{|c|}{ Phase 2 (at. \%) } & \multicolumn{3}{|c|}{ Phase 3 (at. \%) } \\
\hline & & $\mathrm{Al}$ & $\mathrm{Cu}$ & $\mathrm{Zn}$ & & $\mathrm{Al}$ & $\mathrm{Cu}$ & $\mathrm{Zn}$ & $\mathrm{Al}$ & $\mathrm{Cu}$ & $\mathrm{Zn}$ & $\mathrm{Al}$ & $\mathrm{Cu}$ & $\mathrm{Zn}$ \\
\hline 300_1 & 700 & 14.3 & 48.8 & 36.9 & $\gamma+\gamma^{\prime}$ & 10.5 & 44.2 & 45.3 & 21.0 & 53.5 & 25.5 & - & - & - \\
\hline $325 \_1$ & 700 & 15.1 & 48.6 & 36.3 & $\gamma+\gamma^{\prime}$ & 11.0 & 45.1 & 43.9 & 20.1 & 53.2 & 26.7 & - & - & - \\
\hline $350 \_1$ & 700 & 14.9 & 48.2 & 36.9 & $\gamma+\gamma^{\prime}$ & 11.4 & 47.0 & 41.6 & 18.5 & 52.4 & 29.1 & - & - & - \\
\hline $375 \_1$ & 700 & 16.9 & 50.8 & 32.3 & $\gamma+\gamma^{\prime}$ & 12.2 & 46.7 & 41.1 & 17.8 & 52.4 & 29.8 & - & - & - \\
\hline $400 \_1$ & 648 & 21.0 & 39.0 & 40.0 & $\tau_{c}+\varepsilon_{1}$ & 29.9 & 46.7 & 23.4 & 17.9 & 36.4 & 45.7 & - & - & - \\
\hline $400 \_2$ & 648 & 42.4 & 52.3 & 5.3 & $\zeta+\eta^{\prime}$ & 45.3 & 51.7 & 3.0 & 41.9 & 52.0 & 6.1 & - & - & - \\
\hline $400 \_3$ & 648 & 14.3 & 61.6 & 24.1 & $\gamma^{\prime}+\beta^{\prime}$ & 22.2 & 61.7 & 16.1 & 10.9 & 61.8 & 27.3 & - & - & - \\
\hline $400 \_4$ & 648 & 53.6 & 42.5 & 3.9 & $\eta^{\prime}+\tau_{r}+\theta$ & 47.8 & 50.2 & 2.0 & 55.3 & 40.0 & 4.7 & 65.4 & 34.0 & 0.6 \\
\hline $400 \_5$ & 648 & 47.6 & 47.7 & 4.8 & $\eta^{\prime}+\tau_{r}$ & 46.59 & 49.61 & 3.80 & 53.1 & 41.0 & 5.9 & - & - & - \\
\hline $400 \_6$ & 648 & 26.1 & 34.8 & 39.1 & $\tau_{\mathrm{i}}+\varepsilon_{1}$ & 42.3 & 42.6 & 15.1 & 13.3 & 30.3 & 56.4 & - & - & - \\
\hline $400 \_7$ & 648 & 18.6 & 42.9 & 38.5 & $\tau_{\mathrm{c}}+\gamma+\varepsilon_{1}$ & 25.6 & 49.1 & 25.3 & 15.7 & 44.4 & 39.9 & 15.4 & 36.8 & 47.8 \\
\hline $400 \_8$ & 648 & 32.8 & 47.8 & 19.4 & $\tau_{c}$ & 32.8 & 47.8 & 19.4 & - & - & - & - & - & - \\
\hline $400 \_9$ & 648 & 37.4 & 46.0 & 16.6 & $\tau_{i}$ & 37.4 & 46.0 & 16.6 & - & - & - & - & - & - \\
\hline 400_10 & 648 & 42.4 & 44.1 & 13.5 & $\tau_{i}$ & 42.4 & 44.1 & 13.5 & - & - & - & - & - & - \\
\hline 400_11 & 625 & 48.4 & 41.7 & 9.9 & $\tau_{r}$ & 48.4 & 41.7 & 9.9 & - & - & - & - & - & - \\
\hline 400_12 & 625 & 29.1 & 57.5 & 13.4 & $\delta+\tau_{c}$ & 29.4 & 59.3 & 11.3 & 28.4 & 50.9 & 20.7 & - & - & - \\
\hline 400_13 & 625 & 38.6 & 55.8 & 5.6 & $\delta+\zeta^{\prime}$ & 35.8 & 59.8 & 4.4 & 41.0 & 52.5 & 6.5 & - & - & - \\
\hline 400_14 & 625 & 15.8 & 49.7 & 34.5 & $\gamma+\gamma^{\prime}$ & 15.8 & 49.2 & 35.0 & 17.4 & 51.0 & 31.6 & - & - & - \\
\hline 400_15 & 625 & 13.8 & 48.2 & 38.0 & $\gamma$ & 13.8 & 48.2 & 38.0 & - & - & - & - & - & - \\
\hline 400_16 & 625 & 11.8 & 46.3 & 41.9 & $\gamma$ & 11.8 & 46.3 & 41.9 & - & - & - & - & - & - \\
\hline 400_17 & 612 & 16.4 & 51.6 & 32.0 & $\gamma^{\prime}$ & 16.4 & 51.6 & 32.0 & - & - & - & - & - & - \\
\hline 400_18 & 612 & 42.9 & 48.3 & 8.8 & $\eta^{\prime}+\tau_{r}$ & 42.9 & 49.6 & 7.5 & 44.7 & 43.8 & 11.5 & - & - & - \\
\hline 400_19 & 612 & 38.2 & 50.7 & 11.1 & $\zeta^{\prime}+\tau_{i}+\delta$ & 40.4 & 50.3 & 9.4 & 34.3 & 48.4 & 17.3 & - & - & - \\
\hline 400_20 & 612 & 31.9 & 58.0 & 10.1 & $\delta+\tau_{c}$ & 32.0 & 59.5 & 8.5 & 30.3 & 51.0 & 18.7 & - & - & - \\
\hline $400 \_21$ & 612 & 28.1 & 40.8 & 31.1 & $\tau_{\mathrm{i}}+\varepsilon_{1}$ & 35.6 & 46.1 & 18.3 & 13.4 & 32.0 & 54.6 & - & - & - \\
\hline 400_22 & 612 & 9.8 & 36.6 & 53.6 & $\gamma+\varepsilon_{1}$ & 9.5 & 40.2 & 50.4 & 10.5 & 32.4 & 57.0 & - & - & - \\
\hline $400 \_23$ & 612 & 5.7 & 55.3 & 39.0 & $\beta$ & 5.7 & 55.3 & 39.0 & - & - & - & - & - & - \\
\hline $400 \_24$ & 612 & 37.5 & 14.8 & 47.7 & $\tau_{\mathrm{r}}+\mathrm{L}$ & 51.1 & 40.2 & 8.7 & 35.0 & 8.6 & 56.4 & - & - & - \\
\hline 400_25 & 660 & 29.4 & 21.8 & 48.8 & $\tau_{\mathrm{r}}+\mathrm{L}+\varepsilon_{1}$ & 50.3 & 40.3 & 9.4 & 31.0 & 8.0 & 61.0 & 8.8 & 23.6 & 67.6 \\
\hline 400_26 & 660 & 56.7 & 4.6 & 38.7 & $\alpha(\mathrm{Al})$ & 56.7 & 4.6 & 38.7 & - & - & - & - & - & - \\
\hline 400_27 & 660 & 38.0 & 34.0 & 28.0 & $\tau_{\mathrm{r}}+\varepsilon_{1}+\mathrm{L}$ & 50.3 & 39.8 & 9.9 & 10.6 & 25.8 & 63.6 & - & - & - \\
\hline $400 \_28$ & 660 & 23.2 & 15.3 & 61.5 & $\varepsilon_{1}+\mathrm{L}$ & 9.96 & 23.12 & 66.92 & 32.3 & 8.1 & 59.6 & - & - & - \\
\hline 400_29 & 660 & 6.1 & 34.3 & 59.6 & $\gamma+\varepsilon_{1}$ & 6.6 & 35.7 & 57.7 & 4.7 & 26.3 & 69.0 & - & - & - \\
\hline 400_30 & 660 & 21.7 & 67.9 & 10.4 & $\gamma^{\prime}+\alpha(\mathrm{Cu})$ & 25.7 & 64.7 & 9.6 & 12.3 & 74.2 & 13.5 & - & - & - \\
\hline 400_31 & 660 & 18.0 & 59.8 & 22.2 & $\gamma^{\prime}+\beta$ & 20.3 & 59.6 & 20.1 & 10.7 & 59.1 & 30.2 & - & - & - \\
\hline $400 \_32$ & 660 & 14.4 & 52.1 & 33.5 & $\gamma^{\prime}$ & 14.4 & 52.1 & 33.5 & & & & - & - & - \\
\hline $400 \_33$ & 660 & 17.0 & 50.2 & 32.8 & $\gamma+\gamma^{\prime}$ & 15.4 & 48.7 & 35.9 & 18.2 & 50.7 & 31.1 & - & - & - \\
\hline $400 \_34$ & 715 & 50.9 & 40.3 & 8.8 & $\tau_{r}$ & 50.9 & 40.3 & 8.8 & & & & - & - & - \\
\hline $400 \_35$ & 715 & 74.8 & 18.8 & 6.4 & $\theta+\alpha(\mathrm{Al})$ & 65.7 & 32.5 & 1.8 & 85.2 & 2.1 & 12.7 & - & - & - \\
\hline $400 \_36$ & 715 & 69.2 & 19.2 & 11.6 & $\theta+\alpha(\mathrm{Al})$ & 65.7 & 31.7 & 2.6 & 78.8 & 3.1 & 18.1 & - & - & - \\
\hline $400 \_37$ & 715 & 26.6 & 6.3 & 67.1 & $\mathrm{~L}$ & 26.5 & 6.3 & 67.2 & & & & - & - & - \\
\hline $400 \_38$ & 715 & 18.6 & 53.7 & 27.7 & $\gamma^{\prime}$ & 18.6 & 53.7 & 27.7 & & & & - & - & - \\
\hline 400_39 & 715 & 63.8 & 18.0 & 18.2 & $\theta+\alpha(\mathrm{Al})$ & 65.8 & 31.5 & 2.7 & 60.3 & 4.1 & 35.4 & - & - & - \\
\hline $400 \_40$ & 715 & 28.8 & 37.8 & 33.4 & $\tau_{\mathrm{i}}+\varepsilon_{1}$ & 40.2 & 43.5 & 16.3 & 13.3 & 31.6 & 55.1 & - & - & - \\
\hline $400 \_41$ & 715 & 59.4 & 20.9 & 19.7 & $\theta+\tau_{\mathrm{r}}+\alpha(\mathrm{Al})$ & 65.8 & 31.9 & 2.3 & 53.5 & 38.7 & 7.8 & 54.0 & 4.7 & 41.3 \\
\hline $400 \_42$ & 680 & 51.1 & 25.1 & 23.8 & $\tau_{\mathrm{r}}+\alpha(\mathrm{Al})+\mathrm{L}$ & 52.7 & 39.0 & 8.3 & 50.3 & 4.7 & 45.0 & 30.0 & 9.8 & 60.2 \\
\hline $400 \_43$ & 680 & 48.6 & 26.7 & 24.7 & $\tau_{\mathrm{r}}+\alpha(\mathrm{Al})+\mathrm{L}$ & 51.7 & 39.9 & 8.4 & 48.7 & 4.6 & 46.7 & 30.3 & 8.5 & 61.2 \\
\hline $400 \_44$ & 680 & 34.1 & 54.8 & 11.1 & $\delta+\zeta^{\prime}+\tau_{c}$ & 32.8 & 60.2 & 7.0 & 38.9 & 51.5 & 9.6 & 32.0 & 50.7 & 17.3 \\
\hline
\end{tabular}


Table 2 continued

\begin{tabular}{|c|c|c|c|c|c|c|c|c|c|c|c|c|c|c|}
\hline \multirow[t]{2}{*}{$\mathrm{T}\left[{ }^{\circ} \mathrm{C}\right] \_$No. } & \multirow[t]{2}{*}{$\begin{array}{l}\text { Annealing time } \\
{[\mathrm{h}]}\end{array}$} & \multicolumn{3}{|c|}{$\begin{array}{l}\text { Overall composition } \\
\text { (at. \%) }\end{array}$} & \multirow[t]{2}{*}{ Coexist. phases } & \multicolumn{3}{|c|}{ Phase 1 (at. \%) } & \multicolumn{3}{|c|}{ Phase 2 (at. \%) } & \multicolumn{3}{|c|}{ Phase 3 (at. \%) } \\
\hline & & $\mathrm{Al}$ & $\mathrm{Cu}$ & $\mathrm{Zn}$ & & $\mathrm{Al}$ & $\mathrm{Cu}$ & $\mathrm{Zn}$ & $\mathrm{Al}$ & $\mathrm{Cu}$ & $\mathrm{Zn}$ & $\mathrm{Al}$ & $\mathrm{Cu}$ & $\mathrm{Zn}$ \\
\hline $400 \_45$ & 680 & 3.5 & 8.3 & 88.2 & $\varepsilon_{1}+\mathrm{L}$ & 1.7 & 15.1 & 83.2 & 4.6 & 3.1 & 92.3 & - & - & - \\
\hline $400 \_46$ & 680 & 30.4 & 49.0 & 20.6 & $\tau_{c}$ & 30.4 & 49.0 & 20.6 & & & & - & - & - \\
\hline $400 \_47$ & 680 & 45.4 & 43.6 & 11.0 & $\tau_{i}$ & 45.4 & 43.6 & 11.0 & & & & - & - & - \\
\hline $400 \_48$ & 680 & 39.9 & 44.6 & 15.5 & $\tau_{i}$ & 39.8 & 44.7 & 15.5 & & & & - & - & - \\
\hline 400_49 & 680 & 35.0 & 46.1 & 18.9 & $\tau_{i}$ & 35.0 & 46.1 & 18.9 & & & & - & - & - \\
\hline 400_50 & 680 & 28.9 & 49.4 & 21.7 & $\tau_{c}$ & 28.9 & 49.4 & 21.7 & & & & - & - & - \\
\hline $450 \_1$ & 500 & 15.9 & 50.9 & 33.2 & $\gamma / \gamma^{\prime}$ & 15.9 & 50.1 & 34.0 & 16.2 & 50.5 & 33.3 & - & - & - \\
\hline $500 \_1$ & 500 & 16.0 & 49.3 & 34.7 & $\gamma / \gamma^{\prime}$ & 15.3 & 52.2 & 32.5 & & & & - & - & - \\
\hline $550 \_1$ & 840 & 32.5 & 55.2 & 12.3 & $\gamma^{\prime}+\tau_{\mathrm{c}}$ & 31.9 & 59.1 & 9.0 & 32.8 & 53.0 & 14.2 & - & - & - \\
\hline $550 \_2$ & 840 & 29.5 & 55.5 & 15.0 & $\gamma^{\prime}+\tau_{c}$ & 29.3 & 57.5 & 13.2 & 30.3 & 52.1 & 17.6 & - & - & - \\
\hline $550 \_3$ & 840 & 21.7 & 55.4 & 22.9 & $\gamma^{\prime}$ & 21.7 & 55.4 & 22.9 & & & & - & - & - \\
\hline $550 \_4$ & 840 & 10.5 & 55.8 & 33.7 & $\beta+\gamma^{\prime}$ & 8.3 & 58.5 & 33.2 & 11.7 & 54.5 & 33.8 & - & - & - \\
\hline $550 \_5$ & 840 & 36.0 & 56.1 & 7.9 & $\delta+\tau_{i}$ & 35.4 & 58.6 & 6.0 & 36.3 & 53.4 & 10.3 & - & - & - \\
\hline $550 \_6$ & 840 & 31.8 & 41.0 & 27.2 & $\tau_{c}+L+\varepsilon_{1}$ & 33.9 & 46.7 & 19.4 & 43.4 & 30.9 & 25.7 & 26.1 & 42.5 & 31.4 \\
\hline $550 \_7$ & 840 & 5.9 & 39.8 & 54.3 & $\gamma$ & 5.9 & 39.8 & 54.3 & - & - & - & - & - & - \\
\hline $550 \_8$ & 840 & 5.4 & 52.5 & 42.1 & $\beta+\gamma^{\prime}$ & 4.5 & 55.2 & 40.3 & 6.6 & 49.0 & 44.4 & - & - & - \\
\hline $550 \_9$ & 840 & 24.3 & 45.5 & 30.2 & $\varepsilon_{1+\gamma}$ & 24.3 & 45.5 & 30.2 & - & - & - & - & - & - \\
\hline 550_10 & 840 & 4.5 & 65.3 & 30.2 & $\alpha(\mathrm{Cu})+\beta$ & 4.1 & 67.0 & 28.9 & 6.2 & 62.3 & 31.5 & - & - & - \\
\hline 550_11 & 840 & 22.6 & 47.4 & 30.0 & $\tau_{\mathrm{c}}+\gamma+\delta$ & 26.6 & 50.0 & 23.4 & 19.2 & 48.6 & 32.2 & 23.9 & 45.4 & 30.7 \\
\hline 550_12 & 420 & 23.7 & 69.9 & 6.4 & $\beta+\gamma^{\prime}$ & 20.4 & 72.6 & 7.0 & 27.6 & 67.0 & 5.4 & - & - & - \\
\hline $550 \_13$ & 420 & 20.2 & 65.9 & 13.9 & $\beta+\gamma^{\prime}$ & 25.1 & 63.5 & 11.4 & 16.2 & 67.6 & 16.2 & - & - & - \\
\hline 550_14 & 420 & 10.2 & 71.3 & 18.5 & $\alpha(\mathrm{Cu})+\beta$ & 9.4 & 72.0 & 18.6 & 13.2 & 67.6 & 19.2 & - & - & - \\
\hline 550_15 & 420 & 40.6 & 48.1 & 11.3 & $\tau_{i}$ & 40.6 & 48.1 & 11.3 & - & - & - & - & - & - \\
\hline 550_16 & 420 & 35.1 & 48.4 & 16.5 & $\tau_{c}$ & 35.1 & 48.4 & 16.5 & - & - & - & - & - & - \\
\hline 550_17 & 420 & 41.3 & 43.8 & 14.9 & $\tau_{\mathrm{i}}+\mathrm{L}$ & 40.6 & 44.4 & 15.0 & 58.7 & 29.5 & 11.8 & - & - & - \\
\hline 550_18 & 420 & 27.5 & 47.1 & 25.4 & $\tau_{\mathrm{c}}+\varepsilon_{1}$ & 29.6 & 49.9 & 20.5 & 26.7 & 46.4 & 26.9 & - & - & - \\
\hline 550_19 & 420 & 30.3 & 34.4 & 35.3 & $\varepsilon_{1}+\mathrm{L}$ & 25.3 & 42.5 & 32.2 & 36.9 & 23.7 & 39.4 & - & - & - \\
\hline 550_20 & 420 & 41.6 & 47.8 & 10.6 & $\tau_{i}$ & 41.6 & 47.8 & 10.6 & - & - & - & - & - & - \\
\hline $550 \_21$ & 560 & 56.8 & 40.3 & 2.9 & $\tau_{\mathrm{r}}+\theta$ & 49.9 & 45.4 & 4.7 & 65.2 & 34.0 & 0.8 & - & - & - \\
\hline 550_22 & 560 & 46.7 & 46.1 & 7.2 & $\tau_{i}$ & 46.7 & 46.1 & 7.2 & - & - & - & - & - & - \\
\hline $550 \_23$ & 560 & 37.4 & 51.9 & 10.7 & $\tau_{i}$ & 37.4 & 51.9 & 10.7 & - & - & - & - & - & - \\
\hline 550_24 & 560 & 12.4 & 46.3 & 41.3 & $\gamma / \gamma^{\prime}$ & 12.4 & 46.3 & 41.3 & - & - & - & - & - & - \\
\hline 550_25 & 560 & 9.5 & 50.1 & 40.4 & $\gamma$ & 9.5 & 50.1 & 40.4 & - & - & - & - & - & - \\
\hline $550 \_26$ & 560 & 9.5 & 47.1 & 43.4 & $\gamma$ & 9.4 & 47.2 & 43.4 & - & - & - & - & - & - \\
\hline 550_27 & 560 & 24.7 & 73.9 & 1.4 & $\alpha(\mathrm{Cu})+\gamma^{\prime}$ & 18.3 & 80.3 & 1.4 & 28.9 & 69.8 & 1.3 & - & - & - \\
\hline 550_28 & 560 & 53.3 & 39.2 & 7.5 & $\tau_{\mathrm{i}}+\mathrm{L}$ & 47.1 & 45.5 & 7.4 & 62.5 & 29.6 & 7.9 & - & - & - \\
\hline 550_29 & 635 & 25.8 & 32.3 & 41.9 & $\varepsilon_{1}+L$ & 21.9 & 39.2 & 38.9 & 31.9 & 19.2 & 48.9 & - & - & - \\
\hline 550_30 & 635 & 25.2 & 49.4 & 25.4 & $\tau_{c}+\gamma^{\prime}$ & 28.2 & 49.8 & 22.0 & 22.7 & 49.1 & 28.2 & - & - & - \\
\hline 550_31 & 635 & 6.2 & 31.5 & 62.3 & $\varepsilon_{1}+\gamma$ & 6.5 & 33.0 & 60.5 & 3.8 & 26.5 & 69.7 & - & - & - \\
\hline 550_32 & 635 & 39.4 & 57.9 & 2.7 & $\delta+\zeta^{\prime}$ & 37.7 & 59.7 & 2.6 & 39.8 & 57.6 & 2.6 & - & - & - \\
\hline 550_33 & 635 & 16.8 & 39.6 & 43.6 & $\gamma+\delta_{1}$ & 16.6 & 41.2 & 42.2 & 17.1 & 39.2 & 43.7 & - & - & - \\
\hline $550 \_34$ & 635 & 42.6 & 54.5 & 2.9 & $\zeta$ & 42.6 & 54.5 & 2.9 & & & & - & - & - \\
\hline 550_35 & 635 & 16.4 & 40.3 & 43.3 & $\gamma+\delta_{1}$ & 15.2 & 42.1 & 42.7 & 16.1 & 39.1 & 44.8 & - & - & - \\
\hline $550 \_36$ & 635 & 3.8 & 30.0 & 66.2 & $\varepsilon_{1}+\gamma$ & 4.2 & 34.9 & 60.9 & 3.9 & 30.7 & 65.4 & - & - & - \\
\hline 550_37 & 635 & 11.6 & 36.1 & 52.3 & $\varepsilon_{1}+\gamma$ & 11.3 & 36.1 & 52.6 & 12.1 & 33.5 & 54.4 & - & - & - \\
\hline $550 \_38$ & 635 & 21.3 & 45.2 & 33.5 & $\gamma+\delta+\varepsilon_{1}$ & 19.1 & 47.4 & 33.5 & 22.0 & 43.4 & 34.6 & - & - & - \\
\hline 700-_1 & 570 & 40.26 & 57.87 & 1.87 & $\varepsilon^{\prime}$ & 40.26 & 57.87 & 1.87 & & & & - & - & - \\
\hline $700 \_2$ & 570 & 38.4 & 56.8 & 4.8 & $\gamma^{\prime}+\varepsilon^{\prime}$ & $*$ & $*$ & $*$ & 38.4 & 56.8 & 4.8 & - & - & - \\
\hline
\end{tabular}


Table 2 continued

\begin{tabular}{|c|c|c|c|c|c|c|c|c|c|c|c|c|c|c|}
\hline \multirow[t]{2}{*}{$\mathrm{T}\left[{ }^{\circ} \mathrm{C}\right]$ No. } & \multirow[t]{2}{*}{$\begin{array}{l}\text { Annealing time } \\
{[\mathrm{h}]}\end{array}$} & \multicolumn{3}{|c|}{$\begin{array}{l}\text { Overall composition } \\
\text { (at. \%) }\end{array}$} & \multirow[t]{2}{*}{ Coexist. phases } & \multicolumn{3}{|c|}{ Phase 1 (at. \%) } & \multicolumn{3}{|c|}{ Phase 2 (at. \%) } & \multicolumn{3}{|c|}{ Phase 3 (at. \%) } \\
\hline & & $\mathrm{Al}$ & $\mathrm{Cu}$ & $\mathrm{Zn}$ & & $\mathrm{Al}$ & $\mathrm{Cu}$ & $\mathrm{Zn}$ & $\mathrm{Al}$ & $\mathrm{Cu}$ & $\mathrm{Zn}$ & $\mathrm{Al}$ & $\mathrm{Cu}$ & $\mathrm{Zn}$ \\
\hline $700 \_3$ & 570 & 33.3 & 59.1 & 7.6 & $\gamma^{\prime}+\varepsilon^{\prime}$ & 33.3 & 59.1 & 7.6 & $*$ & $*$ & $*$ & - & - & - \\
\hline $700 \_4$ & 570 & 32.8 & 56.8 & 10.4 & $\gamma^{\prime}$ & 32.8 & 56.8 & 10.4 & & & & - & - & - \\
\hline $700 \_5$ & 570 & 30.4 & 56.9 & 12.7 & $\gamma+\tau_{c}$ & 29.7 & 59.5 & 10.8 & 30.8 & 56.3 & 12.9 & - & - & - \\
\hline $700 \_6$ & 570 & 27.9 & 57.5 & 14.6 & $\gamma+\tau_{c}$ & 27.7 & 57.7 & 14.6 & $*$ & $*$ & $*$ & - & - & - \\
\hline $700 \_7$ & 570 & 24.4 & 55.7 & 19.9 & $\gamma$ & 24.4 & 55.7 & 19.9 & & & & - & - & - \\
\hline $700 \_8$ & 570 & 17.5 & 54.7 & 27.8 & $\gamma$ & 17.5 & 54.7 & 27.8 & - & - & - & - & - & - \\
\hline $700 \_9$ & 450 & 35.5 & 58.4 & 6.1 & $\gamma^{\prime}+\varepsilon^{\prime}$ & 34.4 & 59.9 & 5.7 & 35.6 & 58.3 & 6.1 & - & - & - \\
\hline 700_10 & 450 & 33.6 & 56.1 & 10.3 & $\gamma^{\prime}+\tau_{c}$ & 32.0 & 58.6 & 9.4 & 33.6 & 55.7 & 10.7 & - & - & - \\
\hline 700_11 & 450 & 13.4 & 73.4 & 13.2 & $\alpha(\mathrm{Cu})+\beta$ & 12.9 & 73.6 & 13.5 & 16.0 & 70.5 & 13.5 & - & - & - \\
\hline 700_12 & 450 & 12.5 & 57.1 & 30.4 & $\beta+\gamma$ & 12.5 & 57.6 & 29.9 & 14.5 & 54.8 & 30.7 & - & - & - \\
\hline 700_13 & 450 & 40.7 & 44.8 & 14.5 & $\tau_{c}+L$ & 34.3 & 51.5 & 14.2 & 45.3 & 39.7 & 15.0 & - & - & - \\
\hline 700_14 & 480 & 33.7 & 43.4 & 22.9 & $\tau_{c}+L$ & 28.9 & 49.0 & 22.1 & 40.9 & 36.5 & 22.6 & - & - & - \\
\hline 700_15 & 480 & 24.8 & 43.0 & 32.2 & $\varepsilon_{1}+L$ & 24.6 & 42.8 & 32.6 & 33.3 & 28.7 & 38.0 & - & - & - \\
\hline 700_16 & 480 & 16.3 & 35.2 & 48.5 & $\varepsilon_{1}+L$ & 16.2 & 39.4 & 44.4 & 16.6 & 28.0 & 55.4 & - & - & - \\
\hline 700_17 & 480 & 12.5 & 32.6 & 54.9 & $\varepsilon_{1}+L$ & 13.0 & 35.6 & 51.4 & 12.6 & 22.3 & 65.1 & - & - & - \\
\hline 700_18 & 480 & 8.1 & 28.2 & 63.7 & $\varepsilon_{1}+L$ & 7.8 & 32.3 & 59.9 & 9.2 & 23.4 & 67.4 & - & - & - \\
\hline 820_1 & 330 & 33.8 & 59.3 & 6.9 & $\gamma^{\prime}+\varepsilon$ & 32.9 & 60.8 & 6.3 & 34.2 & 59.0 & 6.8 & - & - & - \\
\hline $820 \_2$ & 330 & 19.6 & 61.1 & 19.3 & $\gamma$ & 19.6 & 61.1 & 19.3 & - & - & - & - & - & - \\
\hline $820 \_3$ & 330 & 36.5 & 60.3 & 3.2 & $\gamma^{\prime}+\varepsilon^{\prime}$ & 35.2 & 61.7 & 3.1 & 37.2 & 59.6 & 3.2 & - & - & - \\
\hline $820 \_4$ & 330 & 28.6 & 57.9 & 13.5 & $\gamma+L$ & 27.7 & 59.1 & 13.2 & 29.4 & 54.9 & 15.7 & - & - & - \\
\hline $820 \_5$ & 330 & 24.8 & 52.6 & 22.6 & $\gamma+L$ & 24.3 & 53.4 & 22.3 & 25.3 & 51.6 & 23.1 & - & - & - \\
\hline $820 \_6$ & 330 & 17.8 & 70.1 & 12.1 & $\beta$ & 17.8 & 70.1 & 12.1 & - & - & - & - & - & - \\
\hline $820 \_7$ & 330 & 30.0 & 61.6 & 8.4 & $\gamma$ & 30.0 & 61.6 & 8.4 & - & - & - & - & - & - \\
\hline $820 \_8$ & 330 & 24.8 & 71.0 & 4.2 & $\beta+\gamma$ & 25.0 & 70.5 & 4.5 & 28.2 & 67.9 & 3.9 & - & - & - \\
\hline 820_9 & 330 & 17.9 & 64.9 & 17.2 & $\beta$ & 17.9 & 64.9 & 17.2 & - & - & - & - & - & - \\
\hline 820_10 & 330 & 15.2 & 76.9 & 7.9 & $\alpha(\mathrm{Cu})+\beta$ & 14.0 & 77.9 & 8.1 & 17.2 & 74.5 & 8.3 & - & - & - \\
\hline 820_11 & 330 & 30.8 & 57.0 & 12.2 & $\gamma+L$ & 30.8 & 57.6 & 11.6 & 32.2 & 54.1 & 13.7 & - & - & - \\
\hline 820_12 & 330 & 9.1 & 68.6 & 22.3 & $\alpha(\mathrm{Cu})+\beta$ & 7.7 & 70.6 & 21.7 & 9.4 & 66.4 & 24.2 & - & - & - \\
\hline 820_13 & 330 & 4.7 & 53.4 & 41.9 & $\beta$ & 4.7 & 53.4 & 41.9 & - & - & - & - & - & - \\
\hline
\end{tabular}

Coexisting phases found by XRD are in italic font. Coexisting phases established from their compositions are in standard font. $*$ composition has not been measured

Table 3 Temperature of phase transitions measured by DTA

\begin{tabular}{|c|c|c|c|c|c|c|c|}
\hline \multirow[t]{2}{*}{$\mathrm{T}\left[{ }^{\circ} \mathrm{C}\right] \_$No. } & \multicolumn{3}{|c|}{ Nominal comp. (at.\%) } & \multicolumn{4}{|c|}{ Thermal effects (heating) $\left({ }^{\circ} \mathrm{C}\right)$} \\
\hline & $\mathrm{Al}$ & $\mathrm{Cu}$ & $\mathrm{Zn}$ & Liquidus & Solidus & $\begin{array}{l}\text { Transition } \\
\gamma \leftrightarrow \gamma^{\prime}\end{array}$ & other transitions \\
\hline $400 \_38$ & 18.6 & 53.7 & 27.7 & 940.7 & 903.8 & 569.1 & \\
\hline $550 \_3$ & 21.7 & 55.4 & 22.9 & 952.2 & 903.6 & 622.2 & 270.2 \\
\hline $700 \_6$ & 27.9 & 57.5 & 14.6 & 955.0 & 892.0 & 727.8 & \\
\hline $700 \_4$ & 32.8 & 56.8 & 10.4 & $*$ & $*$ & 826.9 & 641.9 \\
\hline
\end{tabular}

*Has not been measured 
binary subsystems, phase rules and data published by Ghosh et al. [1].

Our results generally agree well with the older phase diagrams published by Ghosh et al. [1], and the theoretical phase diagram published by Liang et al. [2], but contains some additional clarification and improvement of areas, which were not investigated in detail in the previous studies.

\section{Isothermal section at $400{ }^{\circ} \mathrm{C}$}

The isothermal section of phase diagram $\mathrm{Al}-\mathrm{Cu}-\mathrm{Zn}$ at $400{ }^{\circ} \mathrm{C}$ is shown in Fig. 2a. The ternary phase field $\tau$ exhibits a long line-shaped homogeneity range between the approximate compositions

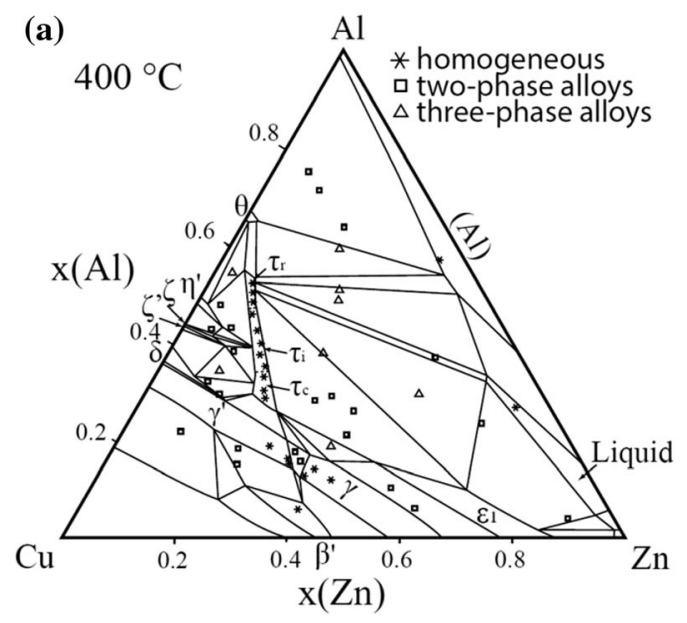

(c)

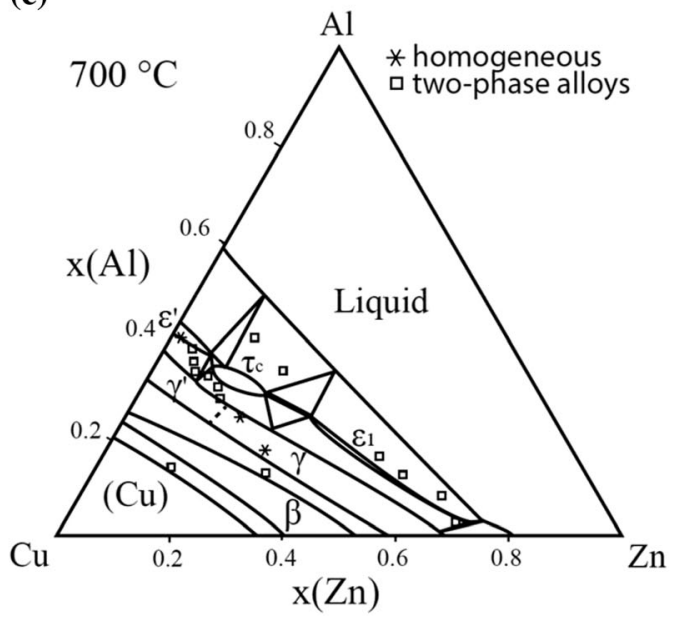

Figure 2 Isothermal sections of the $\mathrm{Al}-\mathrm{Cu}-\mathrm{Zn}$ experimental phase diagram at a $400{ }^{\circ} \mathrm{C}$, b $550{ }^{\circ} \mathrm{C}$, c $700{ }^{\circ} \mathrm{C}$, d $820{ }^{\circ} \mathrm{C}$. Overall compositions of selected samples are represented by several symbols. Stars represent the samples located in the singlephase field. Squares are the overall composition of the samples
$\mathrm{Al} 29 \mathrm{Cu} 49 \mathrm{Zn} 22$ and $\mathrm{Al} 48 \mathrm{Cu} 42 \mathrm{Zn} 10$. In this area, we found at least three structural modifications (cubic form $\tau_{\mathrm{c}}$ (presumably) incommensurate modification $\tau_{\mathrm{i}}$ and rhombohedral $\tau_{\mathrm{r}}$ ). The structural details are described in detail in the following paragraph. The Zn-poor region of the isothermal section shows equilibria of the $\tau$-phase family with various binary $\mathrm{Al}-\mathrm{Cu}$ compounds (phases $\theta, \eta^{\prime}, \zeta, \zeta^{\prime}, \delta$ ). The Al-Cu binary compounds in this central region show limited solubility of $\mathrm{Zn}$. The solubility of $\mathrm{Zn}$ in the $\theta$-phase is about 1 at. $\%$. The phases $\eta^{\prime}, \zeta, \zeta^{\prime}$ show solubility of $\mathrm{Zn}$ about 5 at. $\%$ and $\delta$ phase about 10 at. $\%$. $\gamma^{\prime}$ phase has highest solubility of $\mathrm{Zn}$ up to 35 at.\% of $\mathrm{Zn}$. Based on binary phase diagram $\mathrm{Cu}-\mathrm{Zn}$, the ordered phase $\beta^{\prime}$ is stable at $400{ }^{\circ} \mathrm{C}$. Solubility of $\mathrm{Al}$ is up to $10 \%$. The $\gamma$

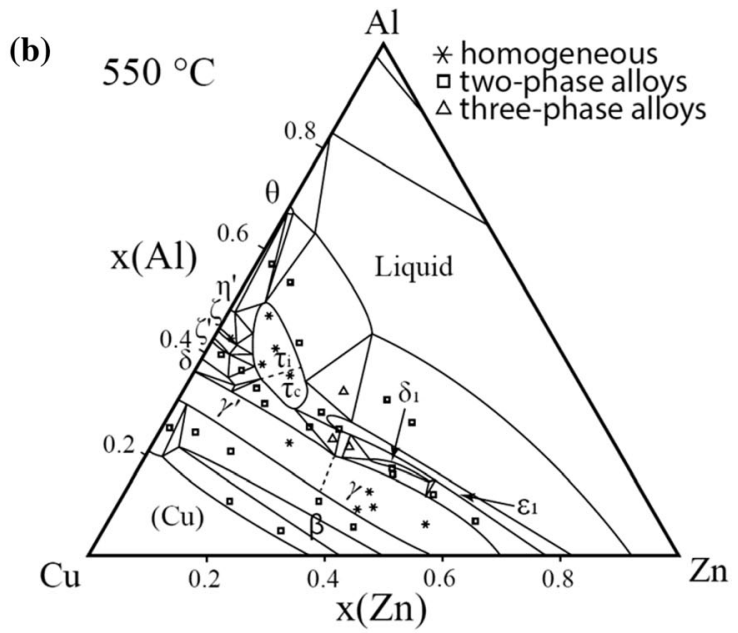

(d)

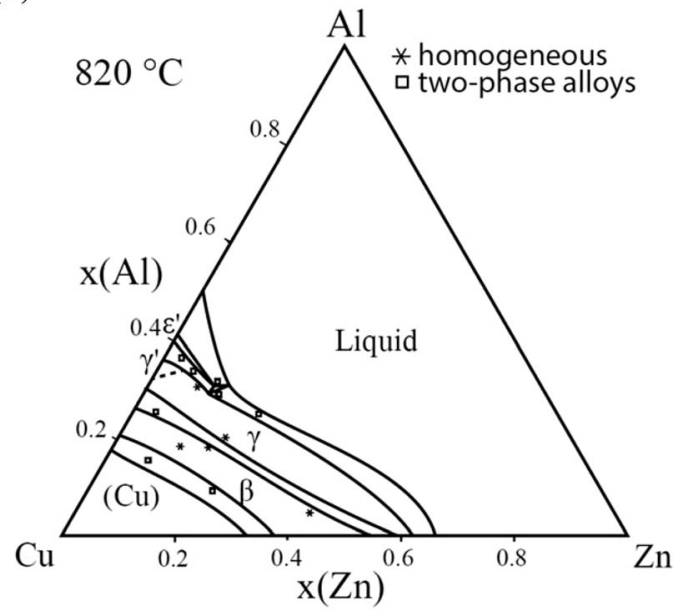

containing two phases in equilibrium. Compositions of each phase and relevant tie lines are not shown as the figure would be very difficult to read. Triangles represent the overall composition of the samples containing three phases in equilibrium. Phase compositions are defined by the corner of the tie triangle. 
phase and $\varepsilon_{1}$ phase have similar solubility of almost 20 at. $\%$ of Al. Figure 3 shows the microstructure of the two-phase sample $\varepsilon_{1}+\tau_{\mathrm{c}}$. The liquid phase is stable around the binary eutectic point in $\mathrm{Al}-\mathrm{Zn}$ and extends toward the more Al-rich compositions in the ternary with a solubility for $\mathrm{Cu}$ up to 5 at.\%. The solubility of $\mathrm{Cu}$ in $\alpha(\mathrm{Al})$ solid solution increases with increasing $\mathrm{Zn}$ content reaches up to 5 at.\%. The microstructure of the $\alpha(\mathrm{Al})$ phase in the two-phase field $\alpha(\mathrm{Al})+\theta$ is not homogeneous (Fig. 4) due to the fact that the annealed samples go through the miscibility gap of $\alpha$ (Al) phase, and it decomposed to two $\alpha$ (Al) phases according the Al-Zn binary phase diagram (Fig. 1b).

\section{The $\tau$-phase}

As mentioned above, the composition area of the $\tau$ phase is crystallographically complex. Originally, two different modifications of the phase were reported in the literature [18]: $\tau$ with CsCl-type structure and $\tau^{\prime}$ with a closely related rhombohedral structure space group $R-3 m$, Pearson symbol $h R 30$. In the latter structure, six atomic sites are present, of which three being occupied by the $\mathrm{Cs}$ atoms and three by the $\mathrm{Cl}$ in specific sublattices of B2. Distortions from the ideal cubic coordination $\mathrm{CN}=14(8+6)$ are only small. Our detailed evaluation of the powder patterns of single-phase samples in the $400{ }^{\circ} \mathrm{C}$ isothermal section yielded the following results: The cubic B2 structure was found in samples situated at the $\mathrm{Cu}$ - and $\mathrm{Zn}$-rich end of the homogeneity range $\left(\tau_{c}\right)$, while the rhombohedral structure was found at the opposite end of the homogeneity range $\left(\tau_{\mathrm{r}}\right)$. The intermediate

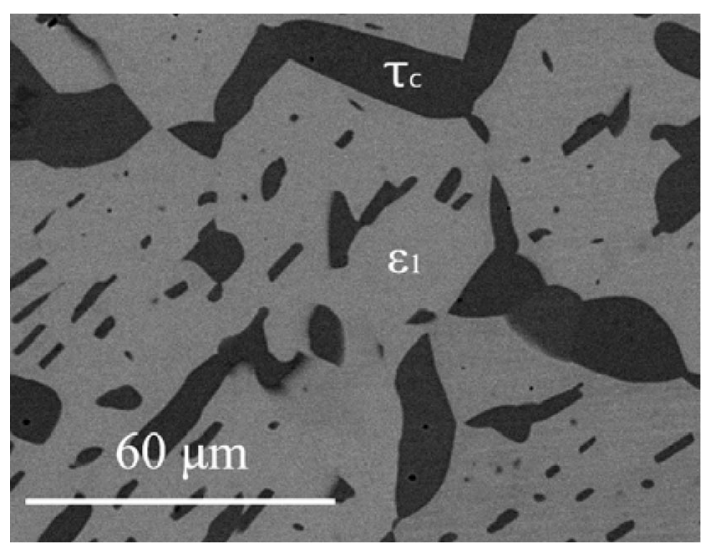

Figure 3 Microstructure of the sample 400_1 in BSE mode consist of $\varepsilon_{1}$ and $\tau_{\mathrm{c}}$ phases.

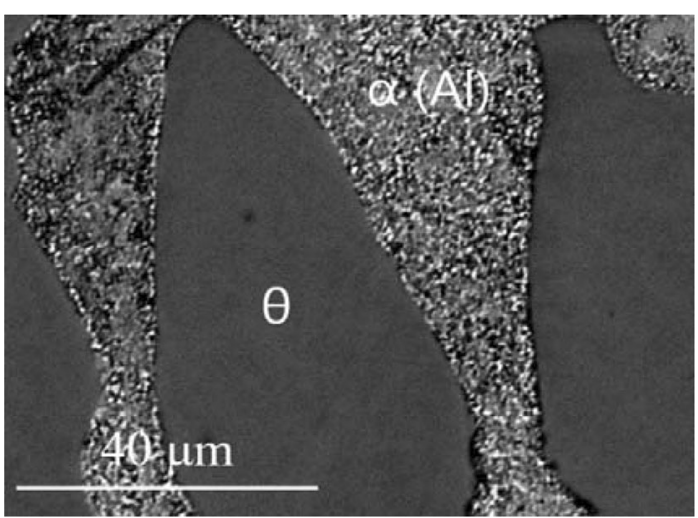

Figure 4 Microstructure of the sample 400_39 in BSE mode consist of $\theta$ and $\alpha(\mathrm{Al})$ phases.

composition range, here designated as $\left(\tau_{\mathrm{i}}\right)$, could not be refined properly, although all samples were single phase according to SEM results. In this area, additional superstructure reflexes were observed which could not be indexed with any reasonable set of cell parameters. Position and intensity of superstructure reflexes varied continuously with the composition, but the number of observed superstructure reflexes increased with decreasing $\mathrm{Cu}$ content. This behavior leads to the conclusion that this intermediate area probably contains an incommensurately modulated crystal structure related to both, the B2 structure and its rhombohedral counterpart. It is not clear if the different phase regions $\tau_{\mathrm{c}}, \tau_{\mathrm{i}}$ and $\tau_{\mathrm{r}}$ are separated by two-phase fields, however, in spite of the large number of samples investigated in this area, it was not possible to identify any composition gap in the phases field. Consequently, the different areas are only separated by dashed lines in Fig. 2a. XRD patterns of some selected samples containing the family of $\tau$ phases are shown in comparison in Fig. 5.

Samples containing $\tau_{\mathrm{c}}$ and $\tau_{\mathrm{r}}$ were further investigated by Rietveld refinement in order to reveal the site occupations. For $\tau_{c}$, a site occupation model allowing vacancies on the $\mathrm{Cu}$ site and $\mathrm{Al} / \mathrm{Zn}$ substitution on the $\mathrm{Al}$ site yielded excellent agreement with the sample composition obtained by EDX measurements (Table 4). This defect mechanism is well in line with other nonstoichiometric B2 intermetallics like e.g., $\mathrm{NiAl}$ showing vacancies on the transition metal site [19].

The rhombohedral structure is much more complicated, so site occupation refinements were more demanding. Table 5 summarizes the refinement results for sample 400_34 in the single-phase region 

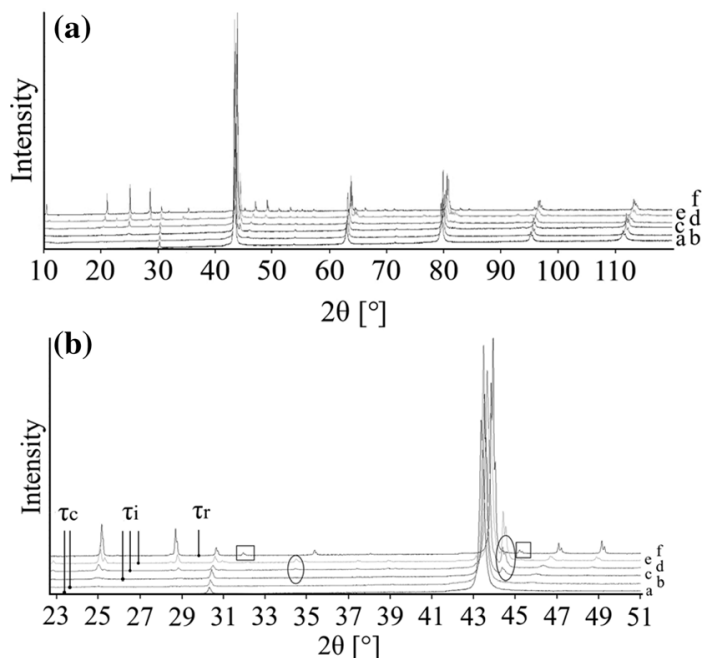

Figure $5 \mathrm{XRD}$ patterns of the $\mathrm{Al}-\mathrm{Cu}-\mathrm{Zn}$ alloy containing $\tau$ phase. a whole measured range, $\mathbf{b}$ central part of the pattern. Specific peaks of $\tau_{\mathrm{i}}$ phase are circled by ellipse. Specific peaks of $\tau_{\mathrm{r}}$ phase are circled by square. Individual patterns were shifted on $\mathrm{Y}$ axe to better visualization. Overall compositions of the samples are following: $a$ : 28.9 at.\% $\mathrm{Al}-\mathrm{Cu}-21.7$ at. $\% \mathrm{Zn}\left(\tau_{\mathrm{c}}\right) ; b: 32.8$ at. $\%$ $\mathrm{Al}-\mathrm{Cu}-19.4$ at.\% $\mathrm{Zn}\left(\tau_{\mathrm{c}}\right) ; c: 35.0$ at.\% Al-Cu-18.9 at.\% $\mathrm{Zn}\left(\tau_{\mathrm{i}}\right) ; d:$ 39.9 at. $\% \mathrm{Al}-\mathrm{Cu}-15.5$ at.\% $\mathrm{Zn}\left(\tau_{\mathrm{i}}\right) ; e 45.4$ at.\% $\mathrm{Al}-\mathrm{Cu}-11.0$ at.\% $\mathrm{Zn}\left(\tau_{\mathrm{i}}\right) ; f 48.4$ at. $\%$ Al-Cu-9.9 at. $\% \mathrm{Zn}\left(\tau_{\mathrm{r}}\right)$.

$\tau_{\mathrm{r}}$. Free refinement of $\mathrm{Cu}$ occupation factors yielded the two fully occupied positions $\mathrm{Cu} 1$ and $\mathrm{Cu} 2$, while the occupation of $\mathrm{Cu} 3$ is only 0.15 . The position $\mathrm{Al} 2$ is fully occupied by $\mathrm{Al}$, while the two positions $\mathrm{All}$ and Al3 show partial substitution with $\mathrm{Zn}$. The resulting calculated overall composition based on Rietveld refinement is in reasonable agreement with results from EDX concentration measurements; a second

Table 4 Comparison of phase compositions for $\tau_{\mathrm{c}}$ and $\tau_{\mathrm{r}}$ determined by Rietveld refinements and EDX

\begin{tabular}{llllll}
\hline Sample & Phase & Method & at.\% Al & at.\% Cu & at.\% Zn \\
\hline $400 \_13$ & \multirow{2}{*}{$\tau_{\mathrm{c}}$} & EDX & 32.7 & 47.8 & 19.5 \\
& & Rietveld & 29.9 & 48.5 & 21.6 \\
$400 \_46$ & \multirow{2}{*}{$\tau_{\mathrm{c}}$} & EDX & 30.4 & 49.0 & 20.6 \\
& & Rietveld & 31.6 & 49.0 & 19.4 \\
$400 \_86$ & \multirow{2}{*}{$\tau_{\mathrm{c}}$} & EDX & 28.9 & 49.5 & 21.6 \\
& & Rietveld & 28.8 & 49.5 & 21.7 \\
$400 \_11$ & \multirow{2}{*}{$\tau_{\mathrm{r}}$} & EDX & 48.4 & 41.7 & 9.9 \\
& & Rietveld & 54.2 & 40.0 & 5.8 \\
$400 \_34$ & \multirow{2}{*}{$\tau_{\mathrm{r}}$} & EDX & 50.9 & 40.3 & 8.8 \\
& & Rietveld & 54.9 & 39.8 & 5.3 \\
\hline
\end{tabular}

sample in the $\tau_{\mathrm{r}}$ region yielded comparable results (Table 4).

\section{Isothermal section at $550{ }^{\circ} \mathrm{C}$}

The isothermal section of phase diagram $\mathrm{Al}-\mathrm{Cu}-\mathrm{Zn}$ at temperature $550{ }^{\circ} \mathrm{C}$ is shown in Fig. $2 \mathrm{~b}$. At this temperature, only $\tau_{\mathrm{C}}$ and $\tau_{\mathrm{i}}$ were identified-the rhombohedral structural modification was not observed. The pseudo-ternary phase $\delta_{1}$ is found at $550{ }^{\circ} \mathrm{C}$ in equilibrium with $\gamma$ (Fig. 6). Thus, the binary $\delta_{1}$ phase in the $\mathrm{Cu}-\mathrm{Zn}$ system is stabilized toward lower temperatures by the addition of Al. Binary Al$\mathrm{Cu}$ compounds (phases $\theta, \eta^{\prime}, \zeta, \zeta^{\prime}, \delta$ ) from the central part of the binary phase diagram are in equilibrium with the $\tau$-phase family with only limited solubility of $\mathrm{Zn}$. This part of the phase diagram is quite complex and phase relations drawn in Fig. $2 b$ are based on diagrams published by Ghosh et al. [1]. The $\theta$ phase shows solubility of $\mathrm{Zn}$ about 1 at.\%. The phases $\eta^{\prime}, \zeta, \zeta^{\prime}, \delta$ show solubility of $\mathrm{Zn}$ about 5 at.\%. The $\gamma^{\prime}$ phase is stable up to 30 at.\% of $\mathrm{Zn}$. At this concentration, a second-order transition between the $\gamma$ and $\gamma^{\prime}$ phases exists. Details of evaluation are discussed together with the vertical section in "The $\gamma / \gamma^{\prime}$ phase field" section. The disordered phase $\beta$ is stable at $550{ }^{\circ} \mathrm{C}$, and it shows high solubility of $\mathrm{Al}$ up to 20 at.\%. $\varepsilon_{1}$ phase has very high solubility at almost 30 at. $\%$ of $\mathrm{Al}$, close to the ternary $\tau_{\mathrm{c}}$. Figure 7 shows the microstructure of a sample in the two-phase field $\tau_{\mathrm{c}}+\varepsilon_{1}$. The liquid phase is stable from Al-Zn binary phase diagram up to 30 at. $\%$ of $\mathrm{Cu}$, and it is connected to the eutectic point of the $\mathrm{Al}-\mathrm{Cu}$ system.

\section{Isothermal section at $700{ }^{\circ} \mathrm{C}$}

The isothermal section of phase diagram $\mathrm{Al}-\mathrm{Cu}-\mathrm{Zn}$ at $700{ }^{\circ} \mathrm{C}$ is presented in Fig. 2c. At this temperature, the cubic modification of $\tau$ phase is the only remaining modification. Figure 8 shows a micrograph of the two-phase field $\tau_{\mathrm{c}}+\mathrm{L}$. The liquid phase is stable from $\mathrm{Cu}-80 \mathrm{Zn}$ to $\mathrm{Al}-40 \mathrm{Cu}$ and covers the whole $\mathrm{Cu}$ poor concentration range. Pseudo-ternary $\varepsilon_{1}$ was found stable between $\gamma$ and the liquid phase. Phase $\varepsilon^{\prime}$ has a solubility up to 5 at. $\%$ of $\mathrm{Zn}$ close to the phase $\tau_{\mathrm{c}}$. The second-order transition between $\gamma$ and $\gamma^{\prime}$ is found around 20 at. $\% \mathrm{Zn}$. The $\beta$ phase exhibits a complete solubility from $\mathrm{Al}-\mathrm{Cu}$ to $\mathrm{Cu}-\mathrm{Zn}$. Figure 9 shows $\beta$ phase in equilibrium with $\gamma$ phase. 
Table 5 Crystallographic parameters for the phase $\tau_{\mathrm{r}}$ in sample 400_34 obtained by Rietveld refinement

\begin{tabular}{|c|c|c|c|c|c|c|}
\hline \multicolumn{7}{|c|}{ Space group $R-3 m$, Pearson symbol $h R 30$} \\
\hline \multicolumn{7}{|c|}{$a=4.126569(18) \AA, c=25.19380(15) \AA$} \\
\hline Site & Wyckoff Pos. & $x$ & $y$ & $z$ & Occupation & $\mathrm{B}_{\mathrm{eq}}$ \\
\hline $\mathrm{Cu} 1$ & $3 a$ & $1 / 3$ & $2 / 3$ & $1 / 8$ & $\mathrm{Cu}: 1.00(14)$ & $0.878(30)$ \\
\hline $\mathrm{Cu} 2$ & $6 c$ & $2 / 3$ & $1 / 3$ & $0.030544(50)$ & $\mathrm{Cu}: 1.00(14)$ & $0.878(30)$ \\
\hline $\mathrm{Cu} 3$ & $6 c$ & 0 & 0 & $0.10673(35)$ & $\mathrm{Cu}: 0.153(20)$ & $0.878(30)$ \\
\hline Al1 & $6 c$ & $1 / 3$ & $2 / 3$ & $0.06919(12)$ & $\begin{array}{l}\text { Al: } 0.90(11) \\
\mathrm{Zn}: 0.10(11)\end{array}$ & $0.678(94)$ \\
\hline $\mathrm{A} 12$ & $6 c$ & $2 / 3$ & $1 / 3$ & $0.132822(92)$ & $\begin{array}{l}\text { Al: } 1.000(94) \\
\mathrm{Zn}: 0.000(94)\end{array}$ & $0.678(94)$ \\
\hline $\mathrm{Al} 3$ & $3 a$ & 0 & 0 & 0 & Al: $0.76(13)$ & $0.678(94)$ \\
\hline
\end{tabular}

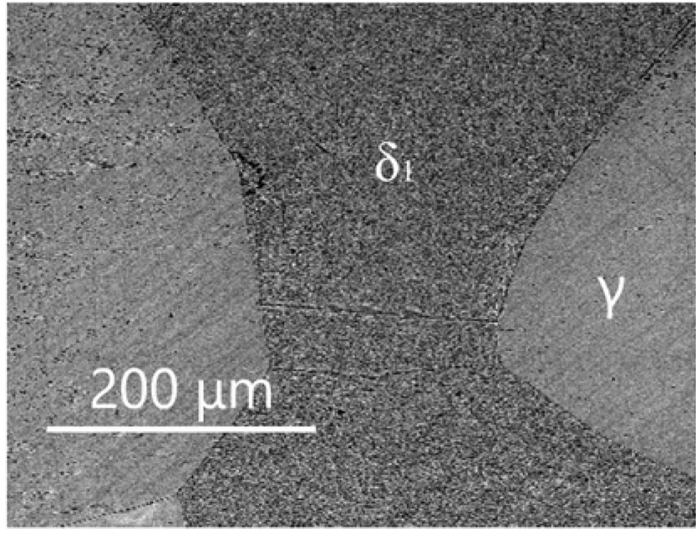

Figure 6 Microstructure of the sample 550_35 in BSE mode consists of $\gamma$ and $\delta_{1}$ phases.

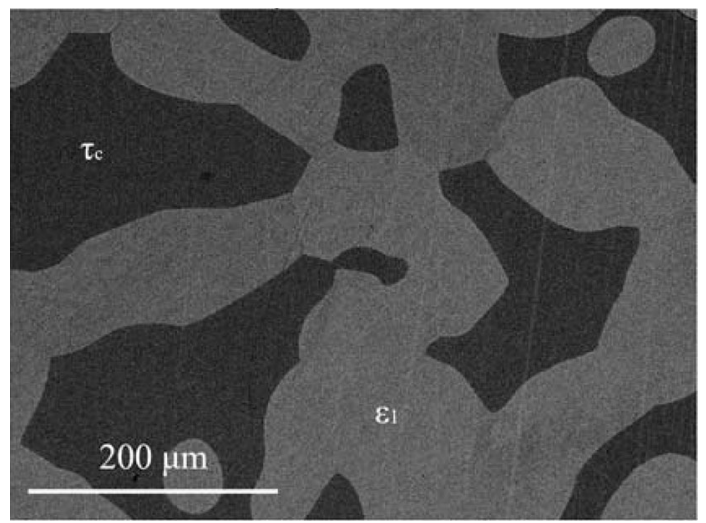

Figure 7 Microstructure of the sample 550_18 in BSE mode consists of $\varepsilon_{1}$ and $\tau_{\mathrm{c}}$ phases.

\section{Isothermal section at $820^{\circ} \mathrm{C}$}

The isothermal section of phase diagram $\mathrm{Al}-\mathrm{Cu}-\mathrm{Zn}$ at $820^{\circ} \mathrm{C}$ is presented in Fig. $2 \mathrm{~d}$. The temperature of $820{ }^{\circ} \mathrm{C}$ was chosen because at this temperature the $\gamma$ phase is stable in $\mathrm{Al}-\mathrm{Cu}$ as well as in the $\mathrm{Cu}-\mathrm{Zn}$

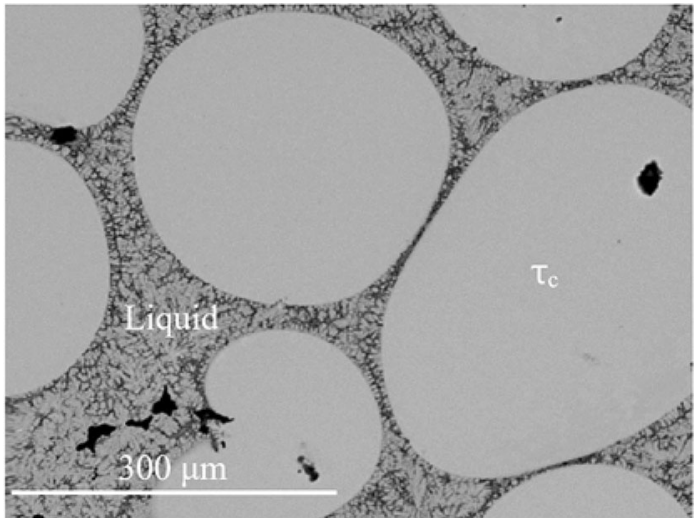

Figure 8 Microstructure of the sample 700 14 in BSE mode consists of liquid and $\tau_{\mathrm{c}}$ phases.

phase diagram. Figure 10 shows the microstructure of a $\gamma+L$ equilibrium. Full mutual solubility between the two binary $\gamma$ phases has been expected and was confirmed. On Al-rich part of $\gamma$-family phase field was found $\gamma^{\prime}$ phase with very low solubility, this is in agreement with binary $\mathrm{Al}-\mathrm{Cu}$ phase diagram.

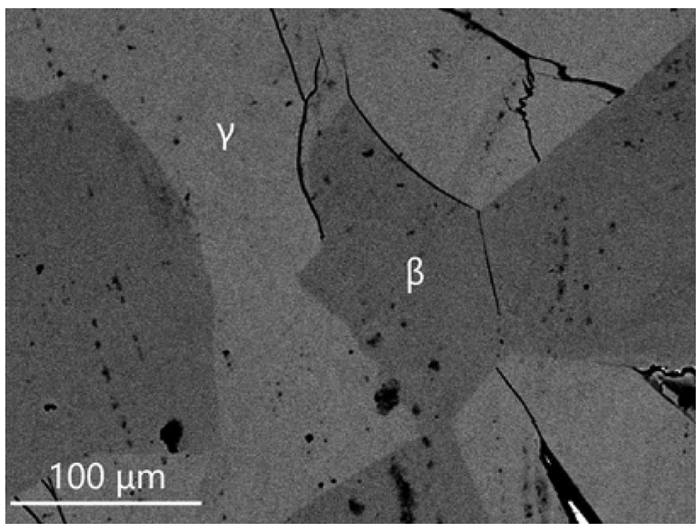

Figure 9 Microstructure of the sample 700_12 in BSE mode consists of $\beta$ and $\gamma$ phases. 
We did not find any ternary or pseudo-ternary phase stable at $820{ }^{\circ} \mathrm{C}$. The $\varepsilon^{\prime}$ phase shows solubility of $\mathrm{Zn}$ up to 15 at.\%, and it is shown in equilibrium with $\gamma^{\prime}$ in Fig. 11.

\section{The $\gamma / \gamma^{\prime}$ phase field}

One of the major goals of the current study was to define the phase equilibrium relations between $\gamma$ (cI52) and $\gamma^{\prime}$ (cP52). As mentioned in the previous sections, a small two-phase field $\gamma+\gamma^{\prime}$ was found at $400{ }^{\circ} \mathrm{C}$, while two different structures were identified in different samples at $550{ }^{\circ} \mathrm{C}$ and $700{ }^{\circ} \mathrm{C}$ (see detailed description below), but no two-phase field was found. In order to define the $\gamma+\gamma^{\prime}$ two-phase field better, a set of additional samples with composition close to the 15 at.\% Al-49 at.\% $\mathrm{Cu}-\mathrm{Zn}$ was prepared and long-term annealed at different temperatures at and below $400{ }^{\circ} \mathrm{C}$. Samples annealed at the temperatures of $300{ }^{\circ} \mathrm{C}, 325^{\circ} \mathrm{C}, 350{ }^{\circ} \mathrm{C}, 375{ }^{\circ} \mathrm{C}$ and $400{ }^{\circ} \mathrm{C}$ show well-crystallized and well-separated two phases $\gamma+\gamma^{\prime}$ in equilibrium (see microstructure on Fig. 12a and XRD pattern Fig. 13a). In contrast to this, samples 15.9 at. $\% \mathrm{Al}-50.9$ at. $\% \mathrm{Cu}-$ $\mathrm{Zn}$ annealed at $450{ }^{\circ} \mathrm{C}, 14.4$ at. $\%$ Al-48.9 at.\% Cu-Zn annealed at $500{ }^{\circ} \mathrm{C}$ and $12.4 \mathrm{Al}-46.3 \mathrm{Cu}-\mathrm{Zn}$ annealed at $550{ }^{\circ} \mathrm{C}$ contained large grains in different orientation (see microstructure on Fig. 12b), but were found to be homogeneous in terms of composition. Their XRD patterns (see e.g., Fig. 13b) showed well-defined diffraction lines of the primitive structure of $\gamma^{\prime}$ in combination with a significant broadening of the basis of all lines fulfilling the reflection conditions for the base-centered structure. Consequently, it was possible to refine the pattern well by assuming an

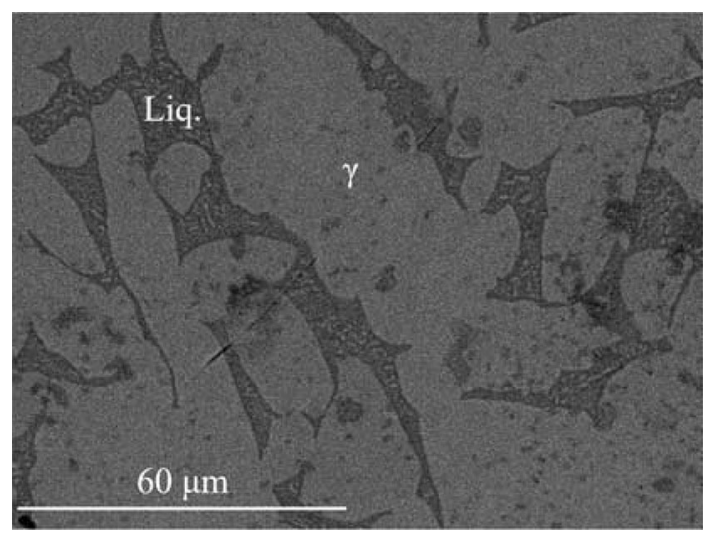

Figure 10 Microstructure of the sample 820_11 in BSE mode consists of liquid and $\gamma$ phases.

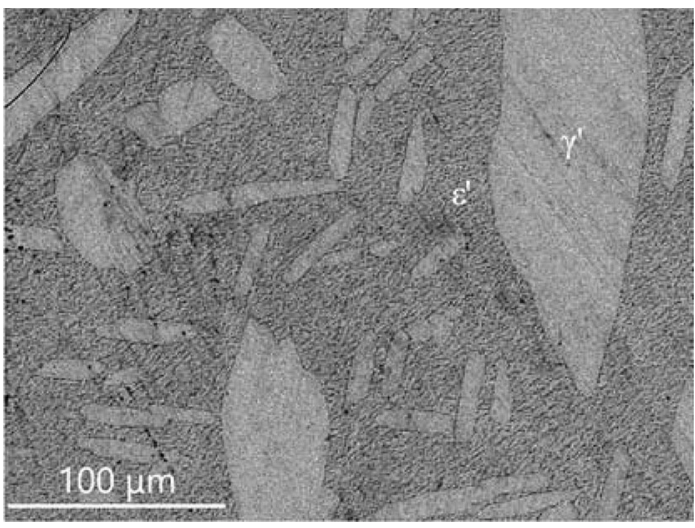

Figure 11 Microstructure of the sample 820_1 in BSE mode consists of $\varepsilon^{\prime}$ and $\gamma^{\prime}$ phases.

overlay of well-crystallized primitive $\gamma^{\prime}$ (refined crystalline domain size $212 \mathrm{~nm}$ ) and base-centered $\gamma$ of poor crystalline quality (refined crystalline domain size $24 \mathrm{~nm}$ ). This leads to the conclusion that these samples were actually single phase at the temperature of annealing and partially transformed during quenching.

This conclusion was actually confirmed by plotting the results from all samples into a vertical section as shown in Fig. 14. The $\gamma^{\prime}+\gamma$ two-phase samples are represented by the tie lines terminated by triangles with overall composition marked by cross symbol. The two-phase gap is getting smaller with increasing temperature and appears to close above $400{ }^{\circ} \mathrm{C}$. Samples showing the characteristically broadened XRD pattern (open squares) are situated in the singlephase region but entered the two-phase field during quenching. Single-phase samples showing $\gamma$ (diamond) or $\gamma^{\prime}$ (full squares) without specific broadening did not show the characteristic line broadening.

In order to confirm the proposed second-order transition line above approximately $440{ }^{\circ} \mathrm{C}$ shown as dashed line in Fig. 14, additional DTA experiments were performed on four samples situated in the respective composition area.

Measurements were performed under a permanent Ar flow of $50 \mathrm{ml} \mathrm{min}^{-1}$ and with heating and cooling rates of $5{ }^{\circ} \mathrm{C} \mathrm{min}{ }^{-1}$. Three runs were performed for each sample; the thermal effects during the first heating run were not taken into account. The temperature of the thermal effects used was thus the average value of the thermal effects of the second and third heating curves only. Small differences between the first and subsequent heating and cooling curves are caused by changes in the shape of the sample 
Figure 12 Micrographs in BSE mode of a sample 350_1 with overall composition 14.9 at. $\% \mathrm{Al}-48.2$ at. $\% \mathrm{Cu}-\mathrm{Zn}$ that had been annealed at $350{ }^{\circ} \mathrm{C}$ containing wellcrystallized phases $\gamma+\gamma^{\prime}$ in equilibriums, b sample 450_1 (15.9 at.\% Al-50.9 at.\% $\mathrm{Cu}-$ $\mathrm{Zn}$ ) annealed at $450{ }^{\circ} \mathrm{C}$ without two-phase structure.
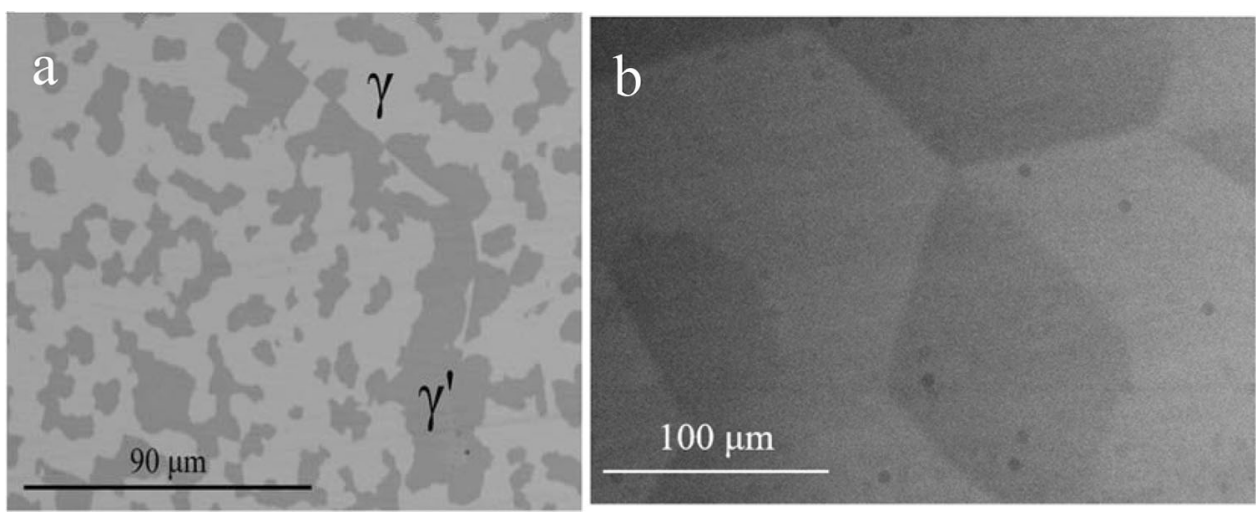
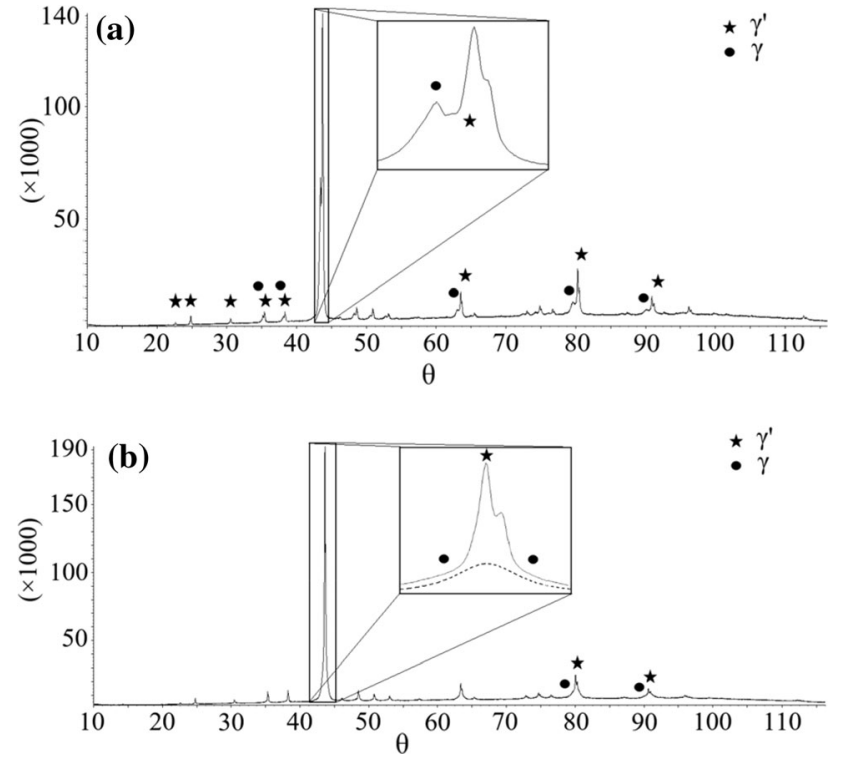

Figure $13 \mathrm{XRD}$ pattern of the alloy $\mathrm{Al}-\mathrm{Cu}-\mathrm{Zn}$ containing. a well-crystallized phases $\gamma$ and $\gamma^{\prime}$ in the sample 14.4 at.\% Al48.8 at. $\% \mathrm{Cu}-\mathrm{Zn}$ annealed at $300{ }^{\circ} \mathrm{C}\left(300 \_1\right)$ and $\mathbf{b}$ poorly crystallized phase $\gamma$ in the sample 15.9 at. $\%$ Al-50.9 at. $\% \mathrm{Cu}-\mathrm{Zn}$ annealed at 450 (450_1).

following initial melting. Temperature of phase transition in solid phase was evaluated as an onset of peak, liquidus was evaluated as a minimum of peak. The results of the DTA third heating and cooling curves of the sample with composition 21.7 at.\% Al55.4 at.\% $\mathrm{Cu}-\mathrm{Zn}$ is presented in Fig. 15. Signals correspond to the temperature of the second-order reaction $\gamma \leftrightarrow \gamma^{\prime}$ at $622.2^{\circ} \mathrm{C}$ (open circles on Fig. 14), to solidus temperature at $903.8^{\circ} \mathrm{C}$ (filled circles on Fig. 14) and to the liquidus temperature at $952.2^{\circ} \mathrm{C}$ (triangles on Fig. 14). Composition of the samples was checked after DTA measurement to ascertain that the sample did not react with the $\mathrm{SiO}_{2}$ material of the ampoules. Thermal analysis results are listed in
Table 3, and the transition temperatures for the $\gamma \leftrightarrow \gamma^{\prime}$ transition are shown as open circles in Fig. 14.

The interpretation of the dashed line separating the $\gamma$ and $\gamma^{\prime}$ phase fields as second-order reaction $\gamma \leftrightarrow \gamma^{\prime}$ is well in line with the observed DTA effects. It also agrees with experimental results from binary phase diagram $\mathrm{Al}-\mathrm{Cu}$, where authors $[3,20]$ proposed a second-order transition between the primitive and base-centered structure there.

\section{Conclusions}

Although the literature related to the $\mathrm{Al}-\mathrm{Cu}-\mathrm{Zn}$ phase diagram is numerous, some of the complex phase equilibria were not well solved. The current study was designed to contribute to a better understanding of those parts of the phase diagram that needed improvement and refinement. The experimental studies were carried out at temperatures $400{ }^{\circ} \mathrm{C}, 550{ }^{\circ} \mathrm{C}, 700{ }^{\circ} \mathrm{C}$ and $820^{\circ} \mathrm{C}$, and some additional measurements were done also at additional temperatures in the $\gamma+\gamma^{\prime}$ phase region. This was achieved by a combination of standard methods: overall and phase compositions of samples were measured using SEM-EDX, the temperatures of phase transitions by DTA measurements in evacuated quartz glass DTA ampoules. The crystal structures were identified by XRD.

The following main results were obtained in present study:

- Mutual relationships of $\gamma+\gamma^{\prime}$ phases were studied in whole concentration and temperature range. Independent two-phase field of $\gamma+\gamma^{\prime}$ was observed up to $400{ }^{\circ} \mathrm{C}$. At higher temperature, the phase transition $\gamma \leftrightarrow \gamma^{\prime}$ is proposed to be second order. 
Figure 14 Vertical section of the ternary phase diagram $\mathrm{Al}-$ $\mathrm{Cu}-\mathrm{Zn}$ between $\mathrm{Al}-0.67 \mathrm{Cu}$ and $\mathrm{Cu}-0.62 \mathrm{Zn}$.
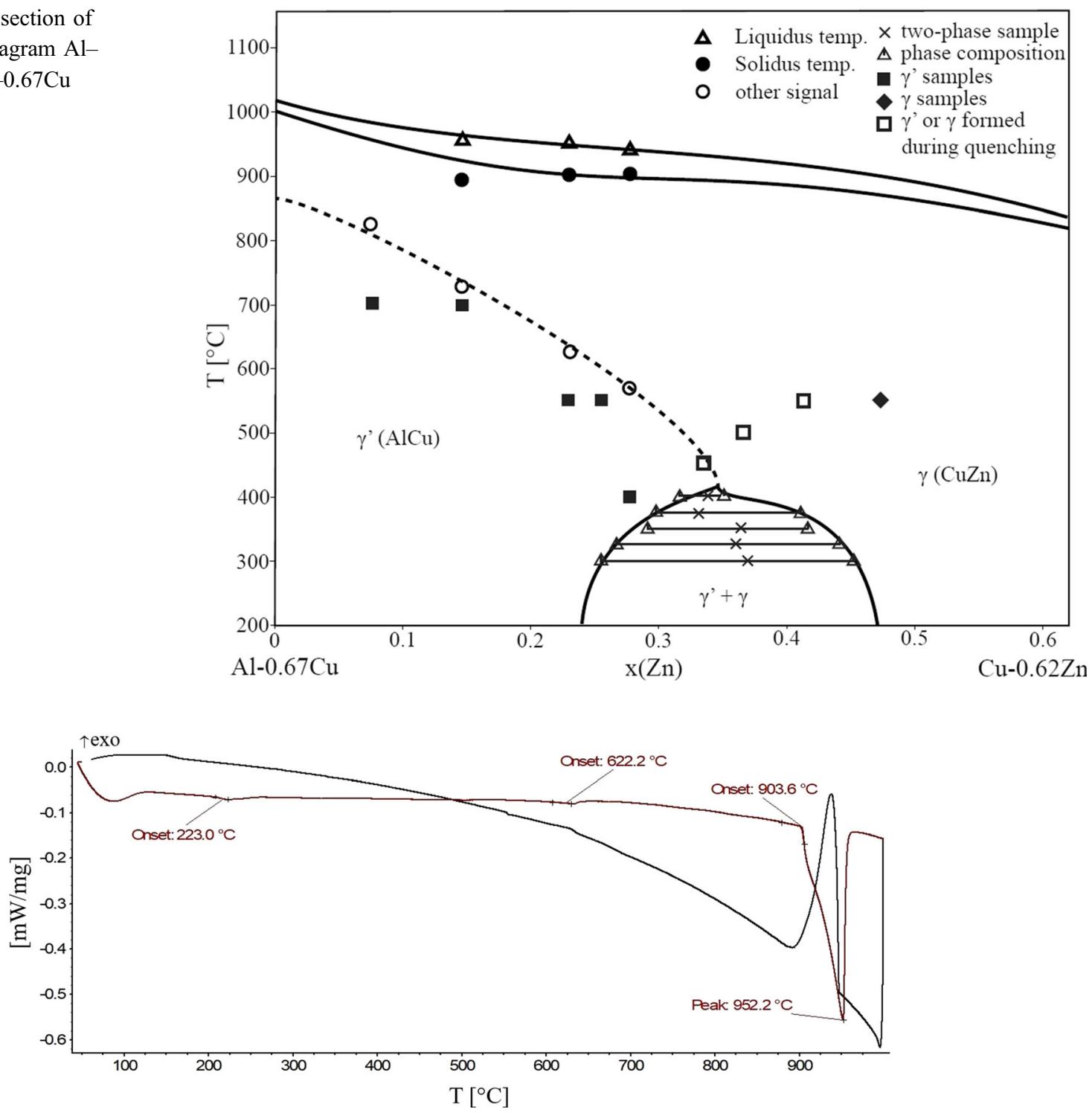

Figure 15 The third DTA heating and cooling curves of the 21.7 at.\% Al-55.4 at.\% $\mathrm{Cu}-\mathrm{Zn}$ sample. Signals correspond to the temperature of the second-order reaction $\gamma \leftrightarrow \gamma^{\prime}\left(632.4{ }^{\circ} \mathrm{C}\right)$ and to

- An isopleth between the binary phases $\gamma$ and $\gamma^{\prime}$ was constructed

- For the ternary phase, at $400{ }^{\circ} \mathrm{C}$ we found strongly temperature-dependent one phase fields containing phases with cubic CsCl-type structure $\left(\tau_{\mathrm{c}}\right)$ and a related rhombohedral structure type $\left(\tau_{\mathrm{r}}\right)$, respectively, and an intermediate composition range with apparently incommensurate modulation $\left(\tau_{\mathrm{i}}\right)$. The rhombohedral structure type $\left(\tau_{\mathrm{r}}\right)$ was not found at $550{ }^{\circ} \mathrm{C}$ and above. At $700{ }^{\circ} \mathrm{C}$ we only found the cubic structure modification $\left(\tau_{\mathrm{r}}\right)$

- Isothermal sections at $400{ }^{\circ} \mathrm{C}, 550{ }^{\circ} \mathrm{C}, 700{ }^{\circ} \mathrm{C}$ and $820{ }^{\circ} \mathrm{C}$ were constructed the liquidus temperature $\left(952.2^{\circ} \mathrm{C}\right)$. Measurement conditions: sample was placed in sealed evacuated quartz glass ampoule, inert atmosphere $5 \mathrm{~N}$ Ar $50 \mathrm{ml} \mathrm{min}{ }^{-1}$, heating rate $5{ }^{\circ} \mathrm{C} \mathrm{min}^{-1}$.

\section{Acknowledgements}

Open access funding provided by Austrian Science Fund (FWF). This study was funded by the Austrian Science Found (FWF) under the Lise-Meitner project M2293-N34. The authors thank Stephan Puchegger from the Department of Dynamic of Condensed Systems at the University of Vienna for the assistance with the SEM measurements and Adela Zemanova from Institute of Physics of Materials, The Czech Academy of Sciences for assistance with some DTA measurement. 


\section{Author contribution}

OZ: Investigation, Resources, Writing - Original Draft, Visualization, Project administration, Funding acquisition. AK: Conceptualization, Methodology, Writing - Review \& Editing. KR: Methodology, Validation, Writing - Review \& Editing, Supervision.

\section{Compliance with ethical standards}

Conflict of interest The authors declare that they have no conflict of interest.

Open Access This article is licensed under a Creative Commons Attribution 4.0 International License, which permits use, sharing, adaptation, distribution and reproduction in any medium or format, as long as you give appropriate credit to the original author(s) and the source, provide a link to the Creative Commons licence, and indicate if changes were made. The images or other third party material in this article are included in the article's Creative Commons licence, unless indicated otherwise in a credit line to the material. If material is not included in the article's Creative Commons licence and your intended use is not permitted by statutory regulation or exceeds the permitted use, you will need to obtain permission directly from the copyright holder. To view a copy of this licence, visit http://creativecommons.org/licen ses/by $/ 4.0 /$.

\section{References}

[1] Ghosh G, van Humbeeck J, Perrot P (2004) Al-Cu-Zn ternary phase diagram evaluation, Ternary Evaluations, MSI, Materials Science. International Services GmbH, Stuttgart

[2] Liang SM, Schmid-Fetzer R (2016) Thermodynamic assessment of the $\mathrm{Al}-\mathrm{Cu}-\mathrm{Zn}$ system, Part III: $\mathrm{Al}-\mathrm{Cu}-\mathrm{Zn}$ ternary system. CALPHAD 52:21-37

[3] Zobac O, Kroupa A, Zemanova A, Richter KW (2019) Experimental description of the Al- $\mathrm{Cu}$ binary phase diagram. Met and Mat. Trans. A 50:3805-3815

[4] Liang SM, Schmid-Fetzer R (2015) Thermodynamic assessment of the $\mathrm{Al}-\mathrm{Cu}-\mathrm{Zn}$ system, part II: Al- $\mathrm{Cu}$ binary system. CALPHAD 51:252-260

[5] Murray JL (1983) The Al-Zn (Aluminum-Zinc) system. Bull Alloy Phase Diagrams 4(1):55-73
[6] Massalski TB (1986) Binary alloy phase diagrams. ASM International, Metals Park, Ohio

[7] Dinsdale AT, Zobac O, Kroupa A, Khvan A (2020) Use of third generation data for the elements to model the thermodynamics of binary alloy systems: Part 1-the critical assessment of data for the Al-Zn system. CALPHAD 68:101723

[8] Dinsdale AT (1991) SGTE data for pure elements. CALPHAD 15(3):317-425

[9] Mathon M, Jardet K, Aragon E, Satre P, Sebaoun A (2000) Al-Ga-Zn system: reassessments of the three binary systems and discussion on possible estimations and on optimisation of the ternary system. CALPHAD 24(3):253-284

[10] A.P. Miodownik, $\mathrm{Cu}-\mathrm{Zn}$ (copper-zinc), published in D.E. Laughlin, D.J. Chakrabarti, P. R. Subramanian: Phase diagrams of binary copper alloys, ASM International, Materials Park OH (1994) 87-496

[11] Liang SM, Hsiao HM, Schmid-Fetzer R (2015) Thermodynamic assessment of the $\mathrm{Al}-\mathrm{Cu}-\mathrm{Zn}$ system, part $\mathrm{I}$ : $\mathrm{Cu}-\mathrm{Zn}$ binary system. CALPHAD 51:224-232

[12] Liang H, Chang YA (1998) A thermodynamic description for the Al-Cu-Zn System. J Phase Equilib 19:25-37

[13] Koster W, Moeller K (1941) Zink-Kupfer-AluminiumLegierungen. Z Metallkde 33:278-283

[14] O. Bauer, M. Hansen, Der Einfluß vondritten Metallen auf die Konstitution der Messinglegierungen, IV. Der Einfluß von Aluminium, Z. Metallkde, 24 (1932) 73-78

[15] Ashirimbetov ZA, Kandaurov NE, Kalina MM, Melikhov VD, Presnyakov AA (1973) Structure and properties of solid solutions of the $\gamma$-region of the $\mathrm{Cu}-\mathrm{Al}-\mathrm{Zn}$ system. Prikl. Teor. Fiz. 5:210-213

[16] J. Gröbner, G. Effenberg Binary evaluations, MSI, Materials Science International Serviced GmbH, Stuttgart, 2004

[17] Topas software, Version 4.2, Bruker, Karlsruhe (2009)

[18] Murphy S (1975) The structure of the T' phase in the system A1-Cu-Zn. Met Sci 9:163-168

[19] Taylor A, Doyle NJ (1972) Further studies on the nickelaluminium system. I. $\beta$-NiAl and $\delta$-Ni2Al3 phase fields. J Appl Cryst 5:201-209

[20] Poweiser N, Lengauer ChL, Richter K (2011) Re-investigation of phase equilibria in the system $\mathrm{Al}-\mathrm{Cu}$ and structural analysis of the high-temperature phase $\eta$-All $-\delta \mathrm{Cu}$. Intermetallics 19:1737-1746

Publisher's Note Springer Nature remains neutral with regard to jurisdictional claims in published maps and institutional affiliations. 